\title{
Campaign Management under Approval-Driven Voting Rules ${ }^{\star}$
}

\author{
Ildikó Schlotter ${ }^{1}$, Piotr Faliszewski ${ }^{2}$, and Edith Elkind ${ }^{3}$ \\ 1 Budapest University of Technology and Economics, Hungary \\ ildi@cs.bme.hu \\ 2 AGH University, Kraków, Poland \\ faliszew@agh.edu.pl \\ 3 University of Oxford, UK \\ elkind@cs.ox.ac.uk
}

\begin{abstract}
Approval-like voting rules, such as Sincere-Strategy Preference-Based Approval voting (SP-AV), the Bucklin rule (an adaptive variant of $k$-Approval voting), and the Fallback rule (a hybrid of the Bucklin rule and SP-AV) have many desirable properties: for example, they are easy to understand and encourage the candidates to choose electoral platforms that have a broad appeal. In this paper, we investigate both classic and parameterized computational complexity of electoral campaign management under such rules. We focus on two methods that can be used to promote a given candidate: asking voters to move this candidate upwards in their preference order or asking them to change the number of candidates they approve of. We show that finding an optimal campaign management strategy of the first type is easy for both Bucklin and Fallback. In contrast, the second method is computationally hard even if the degree to which we need to affect the votes is small. Nevertheless, we identify a large class of scenarios that admit fixed-parameter tractable algorithms.
\end{abstract}

Keywords: approval voting; Bucklin voting; fallback voting; campaign management; bribery; parameterized complexity.

\section{Introduction}

Approval voting - a voting rule that asks each voter to report which candidates she approves of and outputs the candidates with the largest number of approvals - is one of the very few election systems that have a real chance of

\footnotetext{
* Ildikó Schlotter was supported by the Hungarian National Research Fund (OTKA grants no. 108383 and no. 108947). Edith Elkind was supported in part by NRF (Singapore) under Research Fellowship NRF RF2009-08 and by the European Research Council StG 639945. Piotr Faliszewski was supported in part by AGH University of Technology Grant no. 11.11.230.124, by Polish Ministry of Science and Higher Education grant N-N206-378637, and by Foundation for Polish Science's program Homing/Powroty.
} 
replacing Plurality voting in political elections. It has many attractive theoretical properties, and its practical usefulness is supported by the experimental results of Laslier and Van der Straeten [36] and Van der Straeten et al. [41]. Some professional organizations, such as, e.g., the Mathematical Association of America (MAA), Institute for Operations Research and Management Sciences (INFORMS), or Institute of Electrical and Electronics Engineers (IEEE), employ Approval voting to select their leaders. One of the major attractions of Approval voting is that, in contrast to the more standard Plurality voting, under Approval voting the candidates can benefit from running their campaigns in a consensus-building fashion, i.e., by choosing a platform that appeals to a large number of voters.

Nonetheless, Approval voting has certain disadvantages as well. Perhaps the most significant of them is its limited expressivity. Indeed, even a voter that approves of several candidates may like some of them more than others; however, Approval voting does not allow her to express this. Therefore, it is desirable to have a voting rule that operates similarly to Approval, yet takes voters' preference orders into account.

Several such voting rules have been proposed. For instance, the Bucklin rule (also known as the majoritarian compromise) asks the voters to gradually increase the number of candidates they approve of, until some candidate is approved by a majority of the voters. The winners are the candidates that receive the largest number of approvals at this point. In a simplified version of this rule, which is popular in the computational social choice literature $[46,45,19]$, the winners are all candidates that are approved by a majority of the voters in the last round. Under both variants of the Bucklin rule, the common approval threshold is lowered gradually, thus reflecting the voters' preferences. However, this common threshold may move past an individual voter's personal approval threshold, forcing this voter to grant approval to a candidate that she does not approve of. To alleviate this problem, Brams and Sanver [7] have recently introduced a new election system, which they call Fallback voting. This system works similarly to the Bucklin rule, but allows each voter to only approve of a limited number of candidates; its simplified version can be defined similarly to simplified Bucklin voting.

With variants of Approval voting gaining wider acceptance, it becomes important to understand whether various activities associated with running an approval-based electoral campaign are computationally tractable. Such activities can be roughly classified into benign, such as winner determination, and malicious, such as manipulation and control; an ideal voting rule admits polynomialtime algorithms for the benign activities, but not for the malicious ones. However, there is an election-related activity that defies such classification, namely, bribery, or campaign management $[25,17,16,4,9]$. Both of these terms are used for actions that aim to make a given candidate an election winner by means of spending money on individual voters so as to change their preference rankings; these actions can be benign if the money is spent on legitimate activities, such as advertising, or malicious, if the voters are paid to vote non-truthfully. 
Now, winner determination for all approval-based rules listed above is clearly easy, and the complexity of manipulation and especially control under such rules is well understood $[34,3,23,38,20,21,28,30]$; see also a recent survey by Faliszewski and Rothe [31]. Thus, in this paper we focus on algorithmic aspects of electoral campaign management. Following Elkind, Faliszewski, and Slinko [17] and Elkind and Faliszewski [16] (see also [13,9]) who study this problem for a variety of preference-based voting rules, we model the campaign management setting using the framework of shift bribery. Under this framework, each voter $v$ is associated with a cost function $\pi$, which indicates, for each $k>0$, how much it would cost to convince $v$ to promote the target candidate $p$ by $k$ positions in her vote. The briber (campaign manager) wants to make $p$ a winner by spending as little as possible. This framework can be used to model a wide variety of campaign management activities, ranging from one-on-one meetings to phone campaigns to direct mailing, each of which has a per-voter cost that may vary from one voter to another.

Note, however, that in the context of approval-based voting rules, we can campaign in favor of a candidate $p$ even without changing the preference order of any voter. Specifically, if some voter $v$ ranks $p$ in position $k$ and currently approves of $k-1$ candidates, we can try to convince $v$ to lower her approval threshold so that she approves of $p$ as well. Similarly, we can try to convince a voter to be more stringent and withdraw her approval from her least preferred approved candidate; this may be useful if that candidate is $p$ 's direct competitor. Arguably, a voter may be more willing to change her approval threshold than to alter her ranking of the candidates. Therefore, such campaign management tactics may be within the campaign manager's budget, even when she cannot afford the more direct approach discussed in the previous paragraph. We will refer to this campaign management technique as "support bribery"; a variant of this model has been considered by Elkind, Faliszewski, and Slinko [17] (a similar, but somewhat different, variant of this model, namely, extension bribery, was studied by Baumeister et al. [4]).

In this paper, we investigate both campaign management activities discussed above, i.e., shift bribery and support bribery, from an algorithmic perspective. We consider five approval-based voting rules, namely, SP-AV (as introduced by Brams and Sanver [6]; see also [3]), Bucklin (both classic and simplified), and Fallback (both classic and simplified). We show that shift bribery is easy with respect to both variants of the Bucklin rule, as well as both variants of the Fallback rule. The argument for the simplified version of both rules relies on dynamic programming, while for the classic version of these rules we use a more involved flow-based approach. In contrast, support bribery tends to be hard; this holds even if we parameterize this problem by the number of voters to be bribed or the total change in the approval counts, and use very simple bribery cost functions. Nevertheless, we identify a natural class of bribery cost functions for which support bribery is fixed-parameter tractable (FPT) with respect to the latter parameter. Interestingly, some of our hardness results hold even for the case of single-peaked profiles, where one often - though certainly 
not always - expects tractability [43, 11, 29, 8,27]. On the other hand, some of the problems considered in this paper do become easy when the input election can be assumed to be single-peaked: in particular, we describe a good (FPT) approximation algorithm for support bribery under SP-AV for the single-peaked domain.

The rest of this paper is organized as follows. In the next section we formally define our model of elections and the voting systems we study, as well as provide the necessary background on (parameterized) computational complexity. We then present our algorithms for shift bribery (Section 3), followed by hardness results (classic and parameterized) and FPT algorithms for support bribery (Section 4). Section 5 contains our results on support bribery for single-peaked elections. We conclude the paper by presenting directions for future research.

\section{Preliminaries}

We denote the set of all non-negative integers by $\mathbb{Z}^{+}$. An election is a pair $E=(C, V)$, where $C=\left\{c_{1}, \ldots, c_{m}\right\}$ is the set of candidates and $V=\left(v^{1}, \ldots, v^{n}\right)$ is the list of voters. Each voter $v^{i}$ is associated with a preference order $\succ^{i}$, which is a total order over $C$, and an approval count $\ell^{i} \in\{0, \ldots,|C|\}$; voter $v^{i}$ is said to approve of the top $\ell^{i}$ candidates in her preference order. We denote by $\operatorname{rank}(c, v)$ the position of candidate $c$ in the preference order of voter $v: v$ 's most preferred candidate has rank 1 and her least preferred candidate has rank $|C|$. A voting rule is a mapping that given an election $E=(C, V)$ outputs a set $W \subseteq C$ of election winners.

We say that an election $(C, V)$ is single-peaked if there is an order $\triangleright$ of the candidates (called the societal axis) such that each voter's preference order $\succ^{i}$ satisfies the following condition: for every triple of candidates $(a, b, c)$, if $a \triangleright b \triangleright c$ or $c \triangleright b \triangleright a$, then $a \succ^{i} b$ implies $b \succ^{i} c$. Equivalently, an election is single-peaked if there is an order $\triangleright$ over the set of candidates such that for each prefix of each vote, the set of candidates included in this prefix forms an interval with respect to $\triangleright$. Given an election, it is easy to verify if it is single-peaked and, if so, to compute one of the societal axes for it in polynomial time $[2,12,24]$. (Interestingly, deciding if a profile is in some sense close to being single-peaked is typically an NP-hard task $[22,10]$, unless we know the axis with respect to which we measure the closeness [27]). Intuitively, the notion of single-peakedness captures scenarios where the electorate is focused on a single one-dimensional issue such as, e.g., the left-to-right political spectrum or the military spending.

Voting Rules We now describe the voting rules that will be considered in this paper. In what follows, we denote the number of voters by $n$. Under $k$-Approval each candidate gets one point from each voter that ranks her in top $k$ positions. The $k$-Approval score $s_{k}(c)$ of a candidate $c \in C$ is the total number of points that she gets, and the winners are the candidates with the highest score. The Bucklin rule, which can be thought of as an adaptive version of $k$-Approval, is defined as follows. Given a candidate $c \in C$, let $s_{B}(c)$ denote the smallest value of $k$ such that at least $\left\lfloor\frac{n}{2}\right\rfloor+1$ voters rank $c$ in the top $k$ positions; we say that $c$ wins 
in round $s_{B}(c)$. The quantity $k_{B}=\min _{c \in C} s_{B}(c)$ is called the Bucklin winning round. Observe that no candidate wins in any of the rounds $\ell<k_{B}$ and at least one candidate wins in round $k_{B}$. The Bucklin winners are the candidates with the highest $k_{B}$-Approval score. Under the simplified Bucklin rule, the winners are the candidates whose $k_{B}$-Approval score is at least $\left\lfloor\frac{n}{2}\right\rfloor+1$; all Bucklin winners are simplified Bucklin winners, but the converse is not necessarily true.

We observe that $k$-Approval, despite its name, ignores the approval counts entirely: a candidate $c$ may fail to get a point from a voter $v^{i}$ who approves of her (if $\ell^{i} \geq \operatorname{rank}\left(c, v^{i}\right)>k$ ), or obtain a point from a voter $v^{j}$ who does not approve of her (if $\ell^{j}<\operatorname{rank}\left(c, v^{j}\right) \leq k$ ). Similarly, neither version of the Bucklin rule uses the information provided by the approval counts. In contrast, the $S P-A V$ rule [6] relies heavily on the approval counts: we define a candidate's approval score to be the number of voters that approve of her, and the winners under SP-AV are the candidates with the highest approval score. Finally, Fallback voting [7] makes use of both the preference orders and the approval counts. Specifically, under this rule we apply the Bucklin rule to the election obtained by deleting each voter's nonapproved candidates from her preference ranking. Since the preference orders are truncated, it may happen that no candidate is ranked by more than half of the voters, in which case the candidates with the highest approval score are elected. We can replace the Bucklin rule with the simplified Bucklin rule in this construction; we will refer to the resulting rule as the simplified Fallback rule.

Parameterized Complexity The framework of parameterized complexity deals with computationally hard problems. In a parameterized problem, each input instance $I$ is associated with an integer $k$ called the parameter, and the aim is to design algorithms that are efficient if the value of the parameter is small. Formally, a problem is said to be fixed-parameter tractable (FPT) with respect to parameter $k$ if it admits an algorithm whose running time on input $(I, k)$ is $f(k)|I|^{O(1)}$ for some computable function $f$, where $|I|$ is the description size of $I$; note that the exponent of $|I|$ does not depend on $k$. Though $f$ is typically an exponential function, such an algorithm is usually more efficient than, for example, one that runs in time $\Theta\left(|I|^{k}\right)$.

Parameterized complexity also has a hardness theory, which relies on parameterized reductions. Given two parameterized problems $Q$ and $Q^{\prime}$, we say that $Q$ admits a parameterized reduction to $Q^{\prime}$ if there is an FPT-computable function $f$ such that for each input $(x, k)$ it holds that $(x, k) \in Q$ if and only if $f(x, k)=\left(x^{\prime}, k^{\prime}\right) \in Q^{\prime}$ and, moreover, $k^{\prime}=g(k)$ for some function $g$. That is, the parameter of the transformed instance only depends on the parameter of the original instance. We call $f$ a parameterized reduction from $Q$ to $Q^{\prime}$.

An analog of the class NP in the parameterized hierarchy is W[1]: a parameterized problem is in $\mathrm{W}[1]$ if it admits a parameterized reduction to the problem of deciding whether a given Turing machine accepts a given input word in at most $k$ steps. The class $\mathrm{W}[2]$ is the next class in the parameterized hierarchy, and we have FPT $\subseteq \mathrm{W}[1] \subseteq \mathrm{W}[2]$. A problem is said to be $\mathrm{W}[1]$-hard (respectively, W[2]-hard), if all problems in W[1] (respectively, W[2]) can be reduced to it by a parameterized reduction. It is conjectured that FPT $\neq \mathrm{W}[1]$. 
Just as NP-hardness of a problem indicates that this problem is unlikely to be polynomial-time solvable, a W[1]-hardness (or, worse yet, W[2]-hardness) result means that the problem (with the given parameterization) is unlikely to admit an FPT algorithm.

To prove $\mathrm{W}[1]$-hardness (or W[2]-hardness) of a parameterized problem $Q$, it suffices to show a parameterized reduction from some parameterized problem already known to be W[1]-hard (respectively, W[2]-hard). In our hardness proofs, we will use the W[1]-hard MUlticolored CLIQUe problem [32] and the W[2]hard DOMINATING SET problem [14].

Definition 1. In the MULTICOLORED CLIQUE problem we are given a graph $G=(\mathcal{V}, \mathcal{E})$, an integer $k$, and a partition of the vertex set $\mathcal{V}$ into $k$ independent sets $\mathcal{V}^{1}, \ldots, \mathcal{V}^{k}$. We ask if $G$ contains a $k$-clique. We take $k$ to be the parameter.

Definition 2. In the DOMINATING SET problem we are given a graph $G$ and a positive integer $k$. We ask if $G$ has a dominating set of size at most $k$, that is, if there exists a subset $S$ of $G$ 's vertices such that (a) $|S| \leq k$, and (b) each vertex not in $S$ has a neighbor in $S$. We take $k$ to be the parameter.

For a more extensive treatment of parameterized complexity, we refer the reader to the excellent textbooks on this subject $[14,40,33]$.

Campaign Management The following definition is adapted from the work of Elkind and Faliszewski [16], which builds on the ideas of Elkind, Faliszewski, and Slinko [17].

Definition 3. Let $\mathcal{R}$ be a voting rule. An instance of $\mathcal{R}$-SHIFT BRIBERY is a tuple $I=(C, V, \Pi, p)$, where $C=\left\{p, c_{1}, \ldots, c_{m-1}\right\}$ is a set of candidates, $V=$ $\left(v^{1}, \ldots, v^{n}\right)$ is a list of voters together with their preference orders over $C$ (and approval counts, if $\mathcal{R}$ uses them), $\Pi=\left(\pi^{1}, \ldots, \pi^{n}\right)$ is a family of cost functions, where each $\pi^{i}$ is a non-decreasing function from $\{0, \ldots,|C|\}$ to $\mathbb{Z}^{+} \cup\{+\infty\}$ that satisfies $\pi^{i}(0)=0$ (each function $\pi^{i}$ is specified by listing its values at $0, \ldots,|C|$ ), and $p \in C$ is a designated candidate. The goal is to find a vector $\mathbf{t}=\left(t_{1}, \ldots, t_{n}\right) \in$ $\left(\mathbb{Z}^{+}\right)^{n}$ with the following properties: (a) if for each $i=1, \ldots, n$ we shift $p$ upwards in the $i$-th vote by $t_{i}$ positions, then $p$ becomes an $\mathcal{R}$-winner of the resulting election, and (b) for all $\mathbf{s}=\left(s_{1}, \ldots, s_{n}\right) \in\left(\mathbb{Z}^{+}\right)^{n}$ that satisfy condition (a) it holds that $\sum_{i=1}^{n} \pi^{i}\left(t_{i}\right) \leq \sum_{i=1}^{n} \pi^{i}\left(s_{i}\right)$. We set $\operatorname{opt}(I)=\sum_{i=1}^{n} \pi^{i}\left(t_{i}\right)$.

In words, $\pi^{i}(k)$ is the cost of shifting the preferred candidate $p$ upwards by $k$ positions in the preferences of the $i$-th voter. A vector $\mathbf{t}=\left(t_{1}, \ldots, t_{n}\right) \in\left(\mathbb{Z}^{+}\right)^{n}$ is called a shift action or simply a (shift) bribery. For any such vector, we denote by $\operatorname{shf}(C, V, \mathbf{t})$ (or by $\operatorname{shf}(E, \mathbf{t})$ ) the election obtained from $E=(C, V)$ by shifting $p$ upwards by $t_{i}$ positions in the $i$-th vote, for each $i=1, \ldots, n$. If $\operatorname{rank}\left(p, v^{i}\right)=k$, but a shift action prescribes shifting $p$ by $k^{\prime} \geq k$ positions in $v^{i}$, we simply place $p$ on top of $v^{i}$. Also, we write $\Pi(\mathbf{t})=\sum_{i=1}^{n} \pi^{i}\left(t_{i}\right)$ to denote the cost of a shift action $\mathbf{t}$.

We say that a shift action $\mathbf{t}=\left(t_{1}, \ldots, t_{n}\right)$ is minimal for $I$, if $\Pi(\mathbf{t})=\operatorname{opt}(I)$, $p$ is a winner in $\operatorname{shf}(C, V, \mathbf{t})$, but for every shift action $\mathbf{s} \neq \mathbf{t}$ such that $s_{i} \leq t_{i}$ 
for all $i=1, \ldots, n$ it holds that $p$ is not a winner in $\operatorname{shf}(C, V, \mathbf{s})$. Note that an optimal shift action is not necessarily minimal, as it may include some shifts of cost zero that are not needed to make $p$ a winner.

Shift bribery does not change the voters' approval counts. A more general notion of bribery, which is relevant for SP-AV and (simplified) Fallback voting, was proposed by Elkind, Faliszewski, and Slinko [17] in the technical report version of their paper [18]. Specifically, they defined mixed bribery for SP-AV, where the briber can both shift the preferred candidate and change the voters' approval counts. In this work, we find it more convenient to separate these two types of bribery. Thus, we will now define support bribery, which focuses on changing the number of approved candidates.

To define support bribery, we need to be able to specify the costs of increasing/decreasing approval counts for the voters. Formally, we assume that each voter $v^{i}$ has a support bribery cost function $\sigma^{i}: \mathbb{Z} \rightarrow \mathbb{Z}^{+} \cup\{+\infty\}$, which satisfies (a) $\sigma^{i}(0)=0$, and (b) for each $k>0$ it holds that $\sigma^{i}(k) \leq \sigma^{i}(k+1)$ and $\sigma^{i}(-k) \leq \sigma^{i}(-k-1)$. For a given $k \in \mathbb{Z}$, we interpret $\sigma^{i}(k)$ as the cost of convincing $v^{i}$ to approve of $\ell^{i}+k$ candidates. By construction, it suffices to define $\sigma^{i}$ on $\left\{-\ell^{i}, \ldots,|C|-\ell^{i}\right\}$, where $\ell^{i}$ is the approval count of $v^{i}$.

Definition 4. Let $\mathcal{R}$ be a voting rule. An instance of $\mathcal{R}$-SUPPORT BRIBERY is a tuple $I=(C, V, \Sigma, p)$, where $C=\left\{p, c_{1}, \ldots, c_{m-1}\right\}$ is a set of candidates, $V=\left(v^{1}, \ldots, v^{n}\right)$ is a list of voters, where each voter $v^{i}$ is represented by her preference order $\succ^{i}$ and her approval count $\ell^{i}$, and $\Sigma=\left(\sigma^{1}, \ldots, \sigma^{n}\right)$ is a family of support bribery cost functions (each function $\sigma^{i}$ is represented by listing its values on $\left.\left\{-\ell^{i}, \ldots,|C|-\ell^{i}\right\}\right)$. The goal is to find a vector $\mathbf{t}=\left(t_{1}, \ldots, t_{n}\right) \in \mathbb{Z}^{n}$ with the following properties: (a) if for each $i=1, \ldots, n$ voter $v^{i}$ changes her approval count from $\ell^{i}$ to $\ell^{i}+t_{i}$, then $p$ is an $\mathcal{R}$-winner of the resulting election, and (b) for all $\mathbf{s}=\left(s_{1}, \ldots, s_{n}\right) \in \mathbb{Z}^{n}$ that satisfy condition (a) it holds that $\sum_{i=1}^{n} \sigma^{i}\left(t_{i}\right) \leq \sum_{i=1}^{n} \sigma^{i}\left(s_{i}\right)$. We set $\operatorname{opt}(I)=\sum_{i=1}^{n} \sigma^{i}\left(t_{i}\right)$.

A vector $\mathbf{t}=\left(t_{1}, \ldots, t_{n}\right) \in \mathbb{Z}^{n}$ is called a push action or a (support) bribery. For any such vector, we denote by $p \operatorname{sh}(C, V, \mathbf{t})$ (or by $p \operatorname{sh}(E, \mathbf{t}))$ the election obtained from election $E=(C, V)$ by setting, for each $i$, the approval count of the $i$-th voter to be $\ell^{i}+t_{i}$. If $\ell^{i}+t_{i}<0$ or $\ell^{i}+t_{i}>m$, then we set the approval count of the $i$-th voter to be 0 or $m$, respectively. We set $\Sigma(\mathbf{t})=\sum_{i=1}^{n} \sigma^{i}\left(t_{i}\right)$.

We say that a push action $\mathbf{t}=\left(t_{1}, \ldots, t_{n}\right)$ is minimal for $I$ if $\Sigma(\mathbf{t})=\operatorname{opt}(I), p$ is a winner in $\operatorname{psh}(C, V, \mathbf{t})$, and for every push action $\mathbf{s} \neq \mathbf{t}$ such that $0 \leq s_{i} \leq t_{i}$ or $t_{i} \leq s_{i} \leq 0$ for all $i=1, \ldots, n$ it holds that $p$ is not a winner in $\operatorname{psh}(C, V, \mathbf{s})$. Note that, as in the case of shift bribery, an optimal push action is not necessarily minimal because it may perform unnecessary zero-cost pushes.

We also consider two natural special types of support bribery cost functions. We say that a support bribery cost function $\sigma$ is positive if $\sigma(k)=+\infty$ for each $k<0$, and we say that it is negative if $\sigma(k)=+\infty$ for each $k>0$. The support bribery problem with positive cost functions corresponds to the setting where the campaign manager can only increase the voters' approval counts, and can be viewed as a fine-grained version of control by adding voters; similarly, 
the support bribery with negative cost functions can be viewed as a refinement of control by deleting voters (see the surveys of Faliszewski, Hemaspaandra, and Hemaspaandra [26] and Faliszewski and Rothe [31] for a discussion of the complexity of election control and for further references).

To conclude this section, we observe that we have defined shift bribery and support bribery as function problems. However, when talking about NPcompleteness, we consider the decision variants of these problems, where we ask if there exists a successful bribery whose total cost does not exceed a given value $b$ - the bribery budget.

\section{Shift Bribery}

In this section, we present our results for SHIFT BRIBERY under the Bucklin rule and the Fallback rule. We start by describing our algorithm for the simplified version of the Bucklin rule; this algorithm can be modified to work for the simplified version of the Fallback rule.

Theorem 1. Simplified Bucklin-SHIFT BRIBERY is in P.

Proof. Given an instance $I=(C, V, \Pi, p)$ of Simplified Bucklin-ShIFT BRIBERY, let $m=|C|, n=|V|$, and let $k$ be the Bucklin winning round for $(C, V)$. Let $W \subseteq C$ be the set of the simplified Bucklin winners in $(C, V)$. We can assume that $p \notin W$.

Let $\mathbf{t}=\left(t_{1}, \ldots, t_{n}\right)$ be a minimal shift action for $I$. Let $\ell$ be the Bucklin winning round in $\operatorname{shf}(C, V, \mathbf{t})$. We claim that $\ell \in\{k, k+1\}$. Indeed, any shift action moves every candidate in $W$ by at most one position downwards in each vote. Therefore, in $\operatorname{shf}(C, V, \mathbf{t})$ all candidates in $W$ win in round $k+1$, and hence $\ell \leq k+1$. Now, suppose that $\ell<k$. In $(C, V)$ the $\ell$-Approval score of every candidate is at most $\left\lfloor\frac{n}{2}\right\rfloor$, so the only candidate that can win in round $\ell$ in $\operatorname{shf}(C, V, \mathbf{t})$ is $p$, and for that she has to be moved into position $\ell$ in some voters' preferences. However, moving $p$ into position $k$ in those voters' preferences suffices to make $p$ a winner in round $k$ (and thus an election winner), and we have assumed that $\mathbf{t}$ is minimal. This contradiction shows that $\ell \geq k$. Hence, to find an optimal shift bribery, it suffices to (a) compute a minimum-cost shift action that makes $p$ a winner in round $k$, (b) compute a minimum-cost shift action that makes $p$ a winner in round $k+1$ and ensures that no other candidate wins in round $k$, and (c) output the cheaper of the two, breaking a tie arbitrarily.

To win in round $k, p$ needs to obtain $\left\lfloor\frac{n}{2}\right\rfloor+1-s_{k}(p)$ additional $k$-Approval points. Thus, to find a minimum-cost shift bribery that makes $p$ win in round $k$, we consider all votes in which $p$ is not ranked in top $k$ positions, order them by the cost of moving $p$ into the $k$-th position (from lowest to highest), and pick the first $\left\lfloor\frac{n}{2}\right\rfloor+1-s_{k}(p)$ of these votes. Let $\mathbf{s}$ denote the shift action that moves $p$ into position $k$ in each of those votes.

Computing a shift action that ensures $p$ 's victory in the $(k+1)$-st round is somewhat more difficult. In this case we need to ensure that (a) each candidate in $W$ is demoted from position $k$ to position $k+1$ enough times that it does not 
win in round $k$, and (b) p's $(k+1)$-Approval score is at least $\left\lfloor\frac{n}{2}\right\rfloor+1$. Thus, we need to find an optimal balance between bribing several groups of voters.

For each $c \in C \backslash\{p\}$, let $V_{c}$ denote the set of all voters that rank $c$ in the $k$-th position and rank $p$ below $c$; note that $c \neq c^{\prime}$ implies $V_{c} \cap V_{c^{\prime}}=\emptyset$. Let us fix a candidate $c$ in $C \backslash\{p\}$. The only way to ensure that $c$ does not win in round $k$ is to shift $p$ into position $k$ in at least $n(c)=\max \left\{0, s_{k}(c)-\left\lfloor\frac{n}{2}\right\rfloor\right\}$ votes in $V_{c}$. Note that $n(c)>0$ if and only if $c \in W$. Thus, if for some $c \in W$ we have $\left|V_{c}\right|<n(c)$, there is no way to ensure that $c$ does not win in round $k$, so in this case we output $\mathbf{s}$ and stop.

Otherwise, we proceed as follows. Let $A_{c}$ be the set of all voters in $V_{c}$ that rank $p$ in position $k+1$, and let $B_{c}=V_{c} \backslash A_{c}$. Note that for each vote in $A_{c}$, shifting $p$ into the $k$-th position does not change the $(k+1)$-Approval score of $p$, while doing the same for a vote in $B_{c}$ increases the $(k+1)$-Approval score of $p$ by one. For each $i=0, \ldots,\left|B_{c}\right|$, let $b(c, i)$ be the minimum cost of a shift action that (a) shifts $p$ into position $k+1$ or above in $i$ votes from $B_{c}$, and (b) shifts $p$ into position $k$ in at least $n(c)$ votes from $A_{c} \cup B_{c}$.

We can compute the numbers $b(c, i)$ for all $c \in C \backslash\{p\}$ and all $i=0, \ldots,\left|B_{c}\right|$ using dynamic programming, as follows. Fix a candidate $c \in C \backslash\{p\}$. Reorder the voters so that the voters in $B_{c}$ appear first, ordered according to their cost of moving $p$ into the $(k+1)$-st position (from lowest to highest), followed by the voters in $A_{c}$, ordered according to their cost of moving $p$ into the $k$-th position (from lowest to highest). After this step the $j$-th voter in $B_{c}$ is $v^{j}$. For each $i$ and $j, 0 \leq i \leq j \leq\left|B_{c}\right|$, and each $h=0, \ldots, n(c)$, we define $b(c, i, j, h)$ to be the cost of a minimum-cost shift action that only involves the voters in $A_{c}$ and the first $j$ voters in $B_{c}$ and that (a) shifts $p$ into position $k+1$ or above in $i$ votes from $B_{c}$, and (b) shifts $p$ into position $k$ in at least $h$ votes from $A_{c} \cup B_{c}$. If there is no such shift action, we set $b(c, i, j, h)=+\infty$.

We can compute $b(c, 0, j, h)$ for all $j=0, \ldots,\left|B_{c}\right|$ and all $h=0, \ldots, n(c)$ by bribing the first $h$ voters in $A_{c}$ to shift $p$ into position $k$. Similarly, we can compute $b(c, i, j, 0)$ for all $0 \leq i \leq j \leq\left|B_{c}\right|$ by bribing the first $i$ voters in $B_{c}$ to shift $p$ into position $k+1$. For all the remaining cases, we compute $b(c, i, j, h)$ using the following formula:

$$
b(c, i, j, h)=\min \left\{\begin{array}{l}
b(c, i-1, j-1, h)+\pi^{j}\left(\operatorname{rank}\left(p, v^{j}\right)-(k+1)\right), \\
b(c, i-1, j-1, h-1)+\pi^{j}\left(\operatorname{rank}\left(p, v^{j}\right)-k\right), \\
b(c, i, j-1, h)
\end{array}\right\} .
$$

The first two lines of this formula correspond to the cases where the $j$-th voter in $B_{c}$ is bribed to shift candidate $p$ into positions $k+1$ and $k$, respectively. The third line deals with the case where this voter is not bribed. It is immediate that this method correctly computes the desired values. By definition, we have $b(c, i)=b\left(c, i,\left|B_{c}\right|, n(c)\right)$. For each candidate $c \in C \backslash\{p\}$ and each $i=0, \ldots,\left|B_{c}\right|$, we define $\mathbf{r}(c, i)$ to be the shift action corresponding to the value $b(c, i)$; this shift action can be extracted from the dynamic programming computation of $b(c, i)$ using standard techniques.

Let $V_{p}=V \backslash \bigcup_{c \in C \backslash p} V_{c}$; the set $V_{p}$ consists of all voters who rank $p$ in their top $k$ positions. Note that in any minimal shift action, voters in $V_{p}$ are not bribed. 
Now, for every $j=1, \ldots, m-1$ and every $i=0, \ldots,\left\lfloor\frac{n}{2}\right\rfloor+1-s_{k+1}(p)$, let $\beta(j, i)$ be the minimum cost of a shift action that (i) only involves voters in $\cup_{\ell=1}^{j} V_{c_{j}}$, (ii) ensures that candidates $c_{1}, \ldots, c_{j}$ do not win in round $k$, and (iii) ensures that $p$ 's $(k+1)$-Approval score is at least $s_{k+1}(p)+i$; we set $\beta(j, i)=+\infty$ if no such shift action exists. The numbers $\beta(j, i), j=1, \ldots, m-1, i=0, \ldots,\left\lfloor\frac{n}{2}\right\rfloor+1-s_{k+1}(p)$, can be computed by dynamic programming as follows. We have

$$
\beta(1, i)= \begin{cases}b\left(c_{1}, i\right) & \text { for } i=0, \ldots, n\left(c_{1}\right), \\ +\infty & \text { for } i=n\left(c_{1}\right)+1, \ldots,\left\lfloor\frac{n}{2}\right\rfloor+1-s_{k+1}(p) .\end{cases}
$$

Further, for every $j>1$ and all $i=0, \ldots,\left\lfloor\frac{n}{2}\right\rfloor+1-s_{k+1}(p)$ we have

$$
\beta(j, i)=\min \left\{\beta(j-1, i-\ell)+b\left(c_{j}, \ell\right) \mid \ell=0, \ldots, \min \left(i, n\left(c_{j}\right)\right)\right\} .
$$

By construction, $\beta\left(m-1,\left\lfloor\frac{n}{2}\right\rfloor+1-s_{k+1}(p)\right)$ is the minimum cost of a shift action ensuring that $p$ wins in round $k+1$ and no other candidate wins in round $k$. A shift action $\mathbf{r}$ that has this cost can be extracted from the dynamic programming computation of $\beta\left(m-1,\left\lfloor\frac{n}{2}\right\rfloor+1-s_{k+1}(p)\right)$; it is the sum of shift actions $\left\{\mathbf{r}\left(c_{j}, i_{j}\right)\right\}_{c_{j} \in C \backslash p}$ for appropriate values of $i_{1}, \ldots, i_{m-1}$.

We output the cheaper of $\mathbf{s}$ and $\mathbf{r}$, breaking a tie arbitrarily. This algorithm runs in polynomial time, and our argument shows that it produces an optimal shift action for $I$.

Theorem 2. Simplified Fallback-SHIFT BRIBERY is in P.

Proof. Given an instance $I=(C, V, \Pi, p)$ of Simplified Fallback-SHIFT BRIBERY, let $m=|C|$ and $n=|V|$. Further, let $\succ^{i}$ be the preference order of voter $v^{i}$, and let $\ell^{i}$ be her approval count. Set $L=\max _{i=1, \ldots, n} \ell^{i}$.

Our algorithm proceeds in two stages. First, it computes a shift action $\mathbf{s}$ of minimum cost that (after deleting the non-approved candidates from each voter's preference list) ensures that there is no winner according to the simplified Bucklin rule and that no candidate has more approvals in total than the preferred candidate $p$. Second, for each $\ell=1, \ldots, L$ it computes a shift action $\mathbf{r}^{\ell}$ of minimum cost ensuring that $p$ wins under simplified Bucklin in round $\ell$ (that is, the Bucklin winning round is $\ell$, and $p$ is an $\ell$-Approval winner). Finally, we output the cheapest among $\mathbf{s}$ and $\mathbf{r}^{\ell}, \ell=1, \ldots, L$, breaking a tie arbitrarily.

In what follows, we say that $p$ can exclude $c$ from round $\ell$ in a vote $v^{i}$ if $c \succ^{i} p$ and $\operatorname{rank}(c, v)=\min \left(\ell^{i}, \ell\right)$. We say that $p$ can exclude $c$ from approval if $c \succ^{i} p$ and $\operatorname{rank}(c, v)=\ell^{i}$.

To obtain $\mathbf{s}$, it suffices to find, for each $t=1, \ldots,\lfloor n / 2\rfloor$, a minimum-cost shift action $\mathbf{s}^{t}$ ensuring that $p$ receives $t$ approvals, and everybody else receives at most $t$ approvals. This can be done greedily, as follows. For each candidate $c \in C \backslash\{p\}$, let $V_{c}$ be the set of votes where $p$ can exclude $c$ from approval. For each $c \in C \backslash\{p\}$ with $s(c)>t$, we order the votes in $V_{c}$ according to their cost $\pi^{i}\left(\operatorname{rank}\left(p, v^{i}\right)-\operatorname{rank}\left(c, v^{i}\right)\right)$, and bribe the cheapest $s(c)-t$ voters in $V_{c}$, breaking ties arbitrarily (if $\left|V_{c}\right|<s(c)-t$ for some such $c$, then $\mathbf{s}^{t}$ remains undefined). If after this stage $p$ has $t^{\prime}<t$ approvals, we bribe the cheapest $t-t^{\prime}$ voters in 
$\bigcup_{C \backslash\{p\}} V_{c}$ that have not been bribed yet, breaking ties arbitrarily (again, if the number of such voters is less than $t-t^{\prime}$, then $\mathbf{s}^{t}$ remains undefined). We then set $\mathbf{s}$ to be the cheapest among $\mathbf{s}^{1}, \ldots, \mathbf{s}^{\lfloor n / 2\rfloor}$, breaking ties arbitrarily (where the cost of an undefined shift action is taken to be $+\infty$ ).

The computation of $\mathbf{r}^{1}$ is easy: we just have to make sure that $p$ receives at least $\lfloor n / 2\rfloor+1$ points in the first round, so we can sort the voters according to the cost of shifting $p$ into the top position (from lowest to highest), and bribe the first $\lfloor n / 2\rfloor+1-s_{1}(p)$ voters. To compute $\mathbf{r}^{\ell}$ for $\ell=2, \ldots, L$, we employ the algorithm used for computing the shift action $\mathbf{r}$ in the proof of Theorem 1, with a few modifications. Specifically, for each $c \in C \backslash\{p\}$ we let $V_{c}$ contain the votes in which $p$ can exclude $c$ from round $\ell-1$. We partition $V_{c}$ into $A_{c}$ and $B_{c}$ by setting $A_{c}=\left\{v^{i} \in V_{c} \mid \operatorname{rank}\left(c, v^{i}\right)=\ell-1, \operatorname{rank}\left(p, v^{i}\right)=\ell \leq \ell^{i}\right\}$ and $B_{c}=V_{c} \backslash A_{c}$. For $i=1, \ldots, n, j=1, \ldots, m$ we say that position $j$ in vote $v^{i}$ is $\ell$-good if $j \leq \min \left(\ell^{i}, \ell\right)$. We then proceed as in the proof of Theorem 1: to determine $\mathbf{r}^{\ell}$, we compute for each $i=0, \ldots,\left|B_{c}\right|$ a minimum-cost shift action that shifts $p$ into an $\ell$-good position in $i$ votes from $B_{c}$ and excludes $c$ from round $\ell-1$ in at least $s_{\ell-1}(c)-\lfloor n / 2\rfloor$ votes from $A_{c} \cup B_{c}$, and then use dynamic programming to decide how many voters from each set $V_{c}, c \in C \backslash\{p\}$, we want to bribe.

Shift bribery also admits a polynomial-time algorithm for the regular version of the Bucklin rule; however, the proof becomes more involved.

Theorem 3. Bucklin-SHIFT BRIBERY is in $\mathrm{P}$.

Proof. Let $m=|C|, n=|V|$, and let $k$ be the Bucklin winning round for $(C, V)$. Let $W$ denote the set of Bucklin winners in $(C, V)$.

Let $\mathbf{t}=\left(t_{1}, \ldots, t_{n}\right)$ be a minimal shift action for $I$, and let $\ell$ be the Bucklin winning round in $\operatorname{shf}(C, V, \mathbf{t})$. We have $\ell \in\{k-1, k, k+1\}$. Indeed, the argument in the proof of Theorem 1 shows that $\ell \leq k+1$. Now, suppose that $\ell<k-1$. This means that in $\operatorname{shf}(C, V, \mathbf{t})$ our preferred candidate $p$ wins in round $\ell$. Consider the set of all voters that were requested to shift $p$ into position $\ell$ or higher under $\mathbf{t}$; this set must be non-empty since prior to the bribery $p$ did not win in round $\ell$. If we now demote $p$ into position $k-1$ in those votes, she would still win in round $k-1$. Moreover, since no other candidate wins in round $k-1$ in the original election, $p$ is now the unique winner in round $k-1$ and hence the Bucklin winner, a contradiction with $\mathbf{t}$ being a minimal shift action.

We will now find (a) a minimum-cost shift action that makes $p$ a winner in round $k-1$, (b) a minimum-cost shift action that makes $p$ a winner in round $k$ and ensures that no candidate has a higher $k$-Approval score than $p$, and (c) a minimum-cost shift action that makes $p$ a winner in round $k+1$ and ensures that no other candidate wins in round $k$ or has a higher $(k+1)$-Approval score than $p$. We will then output the cheapest of these three shift actions, breaking ties arbitrarily.

The first step is straightforward: we order all voters that do not rank $p$ in the first $k-1$ positions by the cost of shifting $p$ into position $k-1$ in their votes 
(from the lowest to the highest), and bribe the first $\left\lfloor\frac{n}{2}\right\rfloor+1-s_{k-1}(p)$ of them to move $p$ into position $k-1$ in their votes. Denote this shift action by $\mathbf{s}$.

The second step is somewhat more difficult. Namely, for each $i=\left\lfloor\frac{n}{2}\right\rfloor+$ $1, \ldots, n$, let $\mathbf{r}^{i}$ be a minimum-cost shift action that ensures that $p$ 's $k$-Approval score is at least $i$, and the $k$-Approval score of any other candidate is at most $i$. We can compute $\mathbf{r}^{i}$ as follows.

Recall that $W$ is the set of Bucklin winners in election $(C, V)$. For each candidate $c \in W$, let $V_{c}$ denote the set of all voters that rank $c$ in the $k$-th position and rank $p$ below $c$. To ensure that $c$ 's $k$-Approval score is at most $i$, we need to shift $p$ into position $k$ in at least $s_{k}(c)-i$ such votes. Thus if for some $c \in W$ we have $\left|V_{c}\right|<s_{k}(c)-i$ then we record that for this value of $i$ the shift action $\mathbf{r}^{i}$ is undefined and we set its cost to $+\infty$. Otherwise, we order the votes in each $V_{c}$ by the cost of moving $p$ into the $k$-th position in this vote (from lowest to highest), and bribe the first $s_{k}(c)-i$ voters in each set to move $p$ in the $k$-th position in their votes; denote the corresponding shift action by $\mathbf{r}^{i, 1}$. In the resulting election $\operatorname{shf}\left(C, V, \mathbf{r}^{i, 1}\right)$, no candidate other than $p$ gets more than $i k$-Approval points. Let $s_{k}^{\prime}$ be $p$ 's $k$-Approval score in $\operatorname{shf}\left(C, V, \mathbf{r}^{i, 1}\right)$. If $s_{k}^{\prime} \geq i$, we set $\mathbf{r}^{i}=\mathbf{r}^{i, 1}$. Otherwise, we order the voters that rank $p$ in position $k+1$ or lower in $\operatorname{shf}\left(C, V, \mathbf{r}^{i, 1}\right)$ by the cost of moving $p$ into position $k$ in their preferences (from lowest to highest), and bribe the first $i-s_{k}^{\prime}$ of them to move $p$ into position $k$ in their votes. Denote this bribery by $\mathbf{r}^{i, 2}$, and set $\mathbf{r}^{i}=\mathbf{r}^{i, 1}+\mathbf{r}^{i, 2}$. Finally, let $\mathbf{r}$ be a cheapest shift action among $\mathbf{r}^{\left\lfloor\frac{n}{2}\right\rfloor+1}, \ldots, \mathbf{r}^{n}$.

Now, finding a minimum-cost shift action that makes $p$ win in round $k+1$ and ensures that no other candidate wins in an earlier round or has more $(k+1)$ Approval points than $p$ is yet more difficult. Indeed, we must balance the need to demote the candidates that may win in round $k$ against the need to demote the candidates that may beat $p$ in round $k+1$. Note also that we may need to shift $p$ into position $k$ in some votes in order to lower the $k$-Approval score of its competitors.

We deal with these issues by reducing our problem to that of finding a minimum-cost circulation. Recall that an instance of a minimum-cost circulation problem is given by a directed graph $\mathcal{G}=(\mathcal{V}, \mathcal{E})$, and, for each $(v, w) \in \mathcal{E}$, a lower bound $l(v, w)$ on the flow from $v$ to $w$, an upper bound $u(v, w)$ on the flow from $v$ to $w$ and the cost $c(v, w)$ of a unit of flow from $v$ to $w$. A solution is a feasible flow, i.e., a vector $(f(v, w))_{(v, w) \in \mathcal{E}}$ that satisfies (a) $l(v, w) \leq f(v, w) \leq u(v, w)$ for all $(v, w) \in \mathcal{E}$ and (b) $\sum_{(z, v) \in \mathcal{E}} f(z, v)=\sum_{(v, w) \in \mathcal{E}} f(v, w)$ for any $v \in \mathcal{V}$. The cost of a feasible solution is given by

$$
\sum_{(v, w) \in \mathcal{E}} c(v, w) f(v, w)
$$

An optimal solution is one that minimizes the cost among all feasible solutions. It is well-known that when all costs and capacities are integers, an optimal circulation is integer and can be found in polynomial time (see, e.g., $[15,42]$ ).

Given an instance of our problem, we construct a family of instances of minimum-cost circulation, one for each $i=\max \left\{0,\left\lfloor\frac{n}{2}\right\rfloor+1-s_{k+1}(p)\right\}, \ldots, n$ as 
follows (see Figure 1). For each $i$, our graph $\mathcal{G}^{i}$ models the situation where we bribe exactly $i$ voters ranking $p$ in position $k+2$ or lower. We let $\mathcal{G}^{i}$ consist of six "layers". The first layer consists of a single vertex $S$, and the second layer consists of a single vertex $S^{\prime}$. In the third layer, we have a vertex $U_{h}$ for each candidate $c_{h} \in C \backslash\{p\}$. In the fourth layer, we have a vertex $W_{j}$ for each $j=1, \ldots, n$. In the fifth layer, we have a vertex $Z_{h}$ for each candidate $c_{h} \in C \backslash\{p\}$. The sixth layer consists of a vertex $T$.

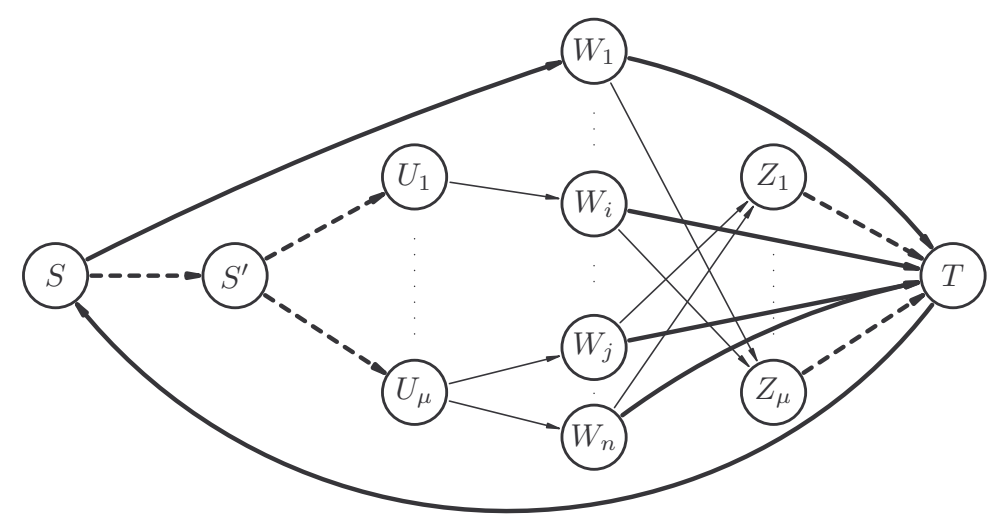

Fig. 1. Graph $\mathcal{G}^{i}$. For readability, we set $\mu=m-1$. The bold arcs are unconstrained, the dashed arcs have a lower bound on the size of the flow, and the regular arcs can carry at most one unit of flow. The graph corresponds to an instance where the voters' preferences are such that $\operatorname{rank}\left(p, v^{1}\right)=\operatorname{rank}\left(c_{1}, v^{i}\right)=\operatorname{rank}\left(c_{m-1}, v^{j}\right)=\operatorname{rank}\left(c_{m-1}, v^{n}\right)=$ $k+1$, and moreover, $\operatorname{rank}\left(c_{m-1}, v^{1}\right)=\operatorname{rank}\left(c_{m-1}, v^{i}\right)=\operatorname{rank}\left(c_{1}, v^{j}\right)=\operatorname{rank}\left(c_{1}, v^{n}\right)=$ $k$.

The costs and capacities of the arcs depend on the value of $i$. We will now describe them layer-by-layer. In our description, we will say that an $\operatorname{arc}(v, w)$ is unconstrained if it satisfies $l(v, w)=0, u(v, w)=+\infty$ and $c(v, w)=0$.

There is an $\operatorname{arc}\left(S, S^{\prime}\right)$ with $l\left(S, S^{\prime}\right)=u\left(S, S^{\prime}\right)=i$ and $c\left(S, S^{\prime}\right)=0$. Also, for each $c_{h} \in C \backslash\{p\}$, there is an arc from $S^{\prime}$ to $U_{h}$ with $l\left(S^{\prime}, U_{h}\right)=$ $\max \left\{0, s_{k+1}\left(c_{h}\right)-s_{k+1}(p)-i\right\}, u\left(S^{\prime}, U_{h}\right)=+\infty$ and $c\left(S^{\prime}, U_{h}\right)=0$.

Each vertex $W_{j}, j=1, \ldots, n$, in the fourth layer has one incoming arc if $v^{j}$ ranks $p$ in position $k+1$ or lower, and no incoming arcs otherwise. Specifically, if $v^{j}$ ranks some $c_{h} \in C \backslash\{p\}$ in position $k+1$, and ranks $p$ in position $k+2$ or lower, then the graph contains an $\operatorname{arc}\left(U_{h}, W_{j}\right)$ with $l\left(U_{h}, W_{j}\right)=0$ and $u\left(U_{h}, W_{j}\right)=1$. We set $c\left(U_{h}, W_{j}\right)$ to be equal to the cost of shifting $p$ into position $k+1$ in $v^{j}$. If $v^{j}$ ranks $p$ in position $k+1$, there is an unconstrained arc from $S$ to $W_{j}$.

There are two types of arcs leaving the vertices in the fourth layer. First, for each $j=1, \ldots, n$, there is an unconstrained $\operatorname{arc}\left(W_{j}, T\right)$. Second, there is an $\operatorname{arc}\left(W_{j}, Z_{h}\right)$ if and only if $v^{j}$ ranks candidate $p$ in position $k+1$ or lower, and $c_{h}$ is ranked in position $k$. We set $l\left(W_{j}, Z_{h}\right)=0, u\left(W_{j}, Z_{h}\right)=1$, and let 
$c\left(W_{j}, Z_{h}\right)$ to be equal to the cost of shifting $p$ from position $k+1$ into position $k$ in the preferences of the $j$-th voter, i.e., $c\left(W_{j}, Z_{h}\right)=\pi^{j}\left(\operatorname{rank}\left(p, v^{j}\right)-k\right)-$ $\pi^{j}\left(\operatorname{rank}\left(p, v^{j}\right)-(k+1)\right)$.

For each vertex $Z_{h}$ in the fifth layer, there is an arc from this vertex to $T$ that satisfies $l\left(Z_{h}, T\right)=\max \left\{0, s_{k}\left(c_{h}\right)-\left\lfloor\frac{n}{2}\right\rfloor\right\}, u\left(Z_{h}, T\right)=+\infty$ and $c\left(Z_{h}, T\right)=0$. Finally, there is an unconstrained $\operatorname{arc}(T, S)$.

In this construction, the flow value on the arc $(T, S)$ corresponds to the number of voters bribed. Specifically, the flow from $S$ to $S^{\prime}$ reflects the number of voters that rank $p$ in position $k+2$ or lower that were bribed to shift $p$ into position $k+1$ or higher, while the flow going directly from $S$ to the vertices in the fourth layer reflects the number of voters that ranked $p$ in position $k+1$ and were bribed to shift $p$ into position $k$. Now, sending $x$ units of flow from $S^{\prime}$ to $U_{h}$ corresponds to shifting $p$ into the $(k+1)$-st position in $x$ votes that rank $c_{h}$ in the $(k+1)$-st position. The lower bound on the flow ensures that we bribe at least $s_{k+1}\left(c_{h}\right)-s_{k+1}(p)-i$ such voters to move $p$ in position $k+1$ or higher, and hence after the bribery $c_{h}$ 's $(k+1)$-Approval score is at most $s_{k+1}(p)+i$. Note that if we bribe exactly $i$ voters that do not rank $p$ in the top $k+1$ positions to shift $p$ into position $k+1$ or higher, $p$ 's $(k+1)$-Approval score becomes exactly $s_{k+1}(p)+i$, so these arcs ensure that no candidate has a higher $(k+1)$-Approval score than $p$.

The arcs between the third and the fourth layer ensure that such a bribery can actually be implemented (i.e., for each $c_{h} \in C \backslash\{p\}$, there are sufficiently many voters that rank $c_{h}$ in the $(k+1)$-st position and rank $p$ below $\left.c_{h}\right)$; their cost reflects the cost of moving $p$ into position $k+1$ in the preferences of the bribed voters.

The flow leaving the fourth layer and going directly into $T$ corresponds to the voters bribed to shift $p$ into position $k+1$ only, while the flow between the fourth and the fifth layer corresponds to the voters that were bribed to move $p$ into position $k$. The cost of the arcs between the fourth and the fifth layer corresponds to the cost of shifting from position $k+1$ to position $k$; note that the cost of shifting $p$ into position $k+1$ in that vote has already been accounted for.

The arcs between the fifth and the sixth layer ensure that no candidate wins in round $k$ : the flow of size $t$ from $Z_{h}$ to $T$ signifies that $t$ voters that rank $c_{h}$ in position $k$ have been bribed to move $p$ into position $k$ (and therefore demote $c_{h}$ into position $k+1$ ). Thus, by satisfying all flow constraints, we ensure that the $k$-Approval score of each candidate in $C \backslash\{p\}$ is at most $\left\lfloor\frac{n}{2}\right\rfloor$.

The argument above implies that every valid circulation in the graph $\mathcal{G}^{i}$, $i=\max \left\{0,\left\lfloor\frac{n}{2}\right\rfloor+1-s_{k+1}(p)\right\}, \ldots, n$, corresponds to a successful shift bribery in which exactly $i$ voters that rank $p$ in position $k+2$ or lower were bribed to shift $p$ into position $k+1$ or higher, and the cost of the circulation is equal to the cost of this bribery. Moreover, the corresponding shift action can be easily computed given the circulation. The converse is also true: if there is a successful shift action of cost $X$ that bribes exactly $i$ voters that rank $p$ in position $k+2$ or lower, there is also a valid circulation of size $i$ that has the same cost. 
For each $i=\max \left\{0,\left\lfloor\frac{n}{2}\right\rfloor+1-s_{k+1}(p)\right\}, \ldots, n$, let $\mathbf{q}^{i}$ denote the shift action that corresponds to an optimal circulation in $\mathcal{G}^{i}$ if one exists; if there is no valid circulation in $\mathcal{G}^{i}$, we leave $\mathbf{q}^{i}$ undefined and set its cost to $+\infty$. Now, let $\mathbf{q}$ be a minimum-cost shift action among $\mathbf{q}^{i}, i=\max \left\{0,\left\lfloor\frac{n}{2}\right\rfloor+1-s_{k+1}(p)\right\}, \ldots, n$; if all $\mathbf{q}^{i}$ are undefined, $\mathbf{q}$ remains undefined as well, and its cost is set to $+\infty$. By construction, $\mathbf{q}$ is a minimum-cost shift action that makes $p$ a winner in the $(k+1)$-st round and ensures that no candidate wins in the $k$-th round or has more $(k+1)$-Approval points than $p$.

Finally, consider the shift actions $\mathbf{s}, \mathbf{r}$, and $\mathbf{q}$, and output one with the minimum cost. This algorithm runs in polynomial time, and outputs a minimum-cost shift action that makes $p$ a winner.

A similar approach works for the Fallback rule.

Theorem 4. Fallback-SHIFT BRIBERY is in $\mathrm{P}$.

Proof. The proof is very similar to that of Theorem 3. We use essentially the same algorithm, but make the following modification. As in the proof of Theorem 2, we compute the cost of a shift bribery that ensures that no candidate is approved by more than half of the voters and our candidate $p$ wins by approval count (if such a shift bribery exists). Then, we apply the algorithm from Theorem 3, taking into account that under Fallback voting it is sometimes possible to ensure that some candidate $c$ becomes disapproved after $p$ is shifted into her position. Modifying the algorithm from Theorem 3 to take advantage of this possibility is straightforward, and we omit a detailed argument.

We remark that Theorem 1 is not a direct consequence of Theorem 3. Indeed, it may be the case that $I$ is a "no"-instance of Bucklin-SHIFT BRIBERY, but a "yes"-instance of Simplified Bucklin-SHIFT BRIBERY (i.e., there is a shift action of cost at most $b$ that makes $p$ a winner under Simplified Bucklin, but not under Bucklin). In this case, running an algorithm for Bucklin-SHIFT BRIBERY on $I$ would output "no" instead of identifying a successful shift action of cost at most $b$ with respect to Simplified Bucklin. Similarly, Theorem 2 is not a direct corollary of Theorem 4 .

\section{Support Bribery}

In the technical report version of their work, Elkind et al. [18] prove an NPcompleteness result for mixed bribery under SP-AV. Their proof does not rely on shifting the preferred candidate in the voters' preferences, and therefore applies to support bribery as well, showing that the decision version of SP-AV-SUPPORT BRIBERY is NP-complete. In this section we extend this result to Fallback voting, and explore the parameterized complexity of support bribery under both the simplified and the classic variant of this rule.

Each instance $I$ of support bribery can be associated with the following parameters. First, let $\alpha(I)$ denote the maximum number of bribed voters over all 
minimal briberies that solve $I$ optimally. Second, let $\beta(I)$ and $\beta^{\prime}(I)$ denote, respectively, the maximum and the minimum of $\sum_{i=1}^{n}\left|t_{i}\right|$ over all minimal briberies $\left(t_{1}, \ldots, t_{n}\right)$ that solve $I$ optimally; these parameters describe the total change in the approval counts. Observe that $\beta(I) \geq \beta^{\prime}(I)$ and $\beta(I) \geq \alpha(I)$ for every instance $I$.

We will now demonstrate that support bribery under Fallback voting is computationally hard, even in very special cases. These results, while somewhat disappointing from the campaign management perspective, are hardly surprising. Indeed, we have argued that support bribery can be viewed as a fine-grained version of control by adding/deleting voters, and both of these control problems are NP-hard for Fallback voting [20]. In fact, since Fallback voting defaults to Approval voting if no candidate is approved by a majority of voters, by introducing appropriate dummy candidates and voters we can easily reduce the problem of control by adding voters under Approval to the problem of support bribery under Fallback voting.

Our next result shows that SUPPORT BRIBERY is NP-hard for both simplified Fallback voting and regular Fallback voting, even under very strong restrictions on the cost function; moreover, these problems remain intractable even for instances with a small value of $\alpha$. Thus, bribing even a few voters can be a hard task.

Theorem 5. Both Fallback-SUPPORT BRIBERY and simplified Fallback-SUPPORT BRIBERY are $\mathrm{NP}$-complete, and also $\mathrm{W}[2]$-hard with respect to parameter $\alpha$ (the maximum number of voters to be bribed), even in the special case where each cost is either $+\infty$ or 0 , and either all cost functions are positive or all cost functions are negative.

Proof. For both types of cost functions (all-positive and all-negative), we give a polynomial-time computable parameterized reduction from the W[2]-hard DOMINATING SET problem; the reductions are inspired by those given by Erdélyi et al. [20] in their proof of $\mathrm{W}[2]$-hardness of control by adding/deleting voters in Fallback voting.

We start by considering negative cost functions. Let $G=(\mathcal{V}, \mathcal{E})$ and $k$ be the given input for DOMINATING SET. We assume $\mathcal{V}=\left\{v_{1}, \ldots, v_{n}\right\}$ and we write $N\left[v_{i}\right]$ to denote the closed neighborhood of vertex $v_{i}$ in $G$, i.e., the set that contains $v_{i}$ and all of its neighbors. We then construct an election $E$ as follows. Our set of candidates is $\mathcal{V} \cup\{a, b, p\} \cup D$, where $p$ is the preferred candidate and $D$ is a set of dummy candidates, $|D|=6 n(n+2)$. There are $6 n$ voters, and each of them approves $n+3$ candidates. Specifically, for each $v_{i} \in \mathcal{V}$, we construct two voters, $x_{i}$ and $\bar{x}_{i}$, and we construct additional $4 n$ voters in order to adjust the scores. The preferences of the voters are shown below. We use dots to denote dummies, and we use sets in the lists when their elements can be ordered arbitrarily. We require that each dummy candidate is approved by at most one voter; the size of $D$ is chosen so as to make this possible. The sign $\mid$ indicates the approval count; non-approved candidates are not listed.

$$
\text { voter } x_{i}: \quad a \succ N\left[v_{i}\right] \succ \ldots \quad \succ p \succ b \mid
$$




$$
\begin{array}{llrl}
\text { voter } \bar{x}_{i}: & a \succ \mathcal{V} \backslash N\left[v_{i}\right] \succ \ldots & \succ p \succ b \mid \\
2 n+1 \text { voters: } & \mathcal{V} \succ \ldots & \succ b \mid \\
n+k \text { voters: } & a \succ \ldots & \succ p \succ b \mid \\
1 \text { voter: } & \ldots & & \succ p \succ b \mid \\
n-k-2 \text { voters: } \ldots & & \succ b \mid
\end{array}
$$

The cost of decreasing the approval count arbitrarily is 0 for each of the votes in $X=\left\{x_{1}, \ldots, x_{n}\right\}$, and is $+\infty$ for all other votes; our budget is 0 . Note that in election $E$ we have $s_{1}(a)=3 n+k, s_{n+1}\left(v_{i}\right)=3 n+1$ for each $i$, $s_{n+2}(p)=3 n+k+1$, and $s_{n+3}(b)=6 n$.

Let $\mathbf{t}$ be some minimal push bribery for $I$. Note that applying $\mathbf{t}$ must decrease $a$ 's first-round score by at least $k$; otherwise $a$ would be the unique winner of election $p \operatorname{sh}(E, \mathbf{t})$ under Fallback as well as under simplified Fallback. Thus, applying $\mathbf{t}$ sets the approval count to 0 in at least $k$ votes in $X$. This decreases $p$ 's score in round $n+2$ by at least $k$ points as well, so $p$ can have at most $3 n+1$ points in round $n+2$ in $p s h(E, \mathbf{t})$. On the other hand, in round $n+3$ candidate $b$ will have more approvals than $p$ under any bribery of cost 0 , so $p$ can become a winner only if it wins in round $n+2$. Therefore, $p$ 's score in round $n+2$ must remain at least $3 n+1$ in $p \operatorname{sh}(E, \mathbf{t})$, which means that $\mathbf{t}$ must set the approval count to 0 in exactly $k$ votes from $X$. Let $S$ be the set of these votes, and let $\left\{s_{1}, \ldots, s_{k}\right\}$ be the corresponding vertices of $G$. As only voters in $X$ can be bribed within the budget and $\mathbf{t}$ is a minimal push action, it follows that voters not in $S$ are not bribed. Consequently, we have $\alpha(I)=k$.

Now, observe that no matter how $S$ is chosen, $a$ does not win in the first $n+1$ rounds in $p \operatorname{sh}(E, \mathbf{t})$, and $p$ gets a strict majority of votes in round $n+2$. Therefore, $p$ wins in $p \operatorname{sh}(E, \mathbf{t})$ if and only if none of the candidates in $\mathcal{V}$ gets $3 n+1$ points in round $n+1$. This happens if and only if each vertex loses at least one point as a result of the push action $\mathbf{t}$, meaning that the sets $N\left[s_{1}\right], \ldots, N\left[s_{k}\right]$ cover $\mathcal{V}$. Since this occurs if and only if the vertices $s_{1}, \ldots, s_{k}$ form a dominating set, we have proved the correctness of the reduction.

We will now consider positive cost functions. Again, the reduction is from DOMINATING SET. Let $G=(\mathcal{V}, \mathcal{E})$ and $k$ be the input instance; we use the notation defined above. Assume without loss of generality that $k \geq 2$. We construct an election $E$ with candidate set $\mathcal{V} \cup\{a, b, p\}$, where $p$ is the preferred candidate. The set of voters is of size $2 n+2$, including a voter $x_{i}$ for each $v_{i} \in \mathcal{V}$. Preferences and approval counts are shown below; we omit the non-approved candidates in the last $n+2$ votes.

voter $x_{i}$ with 0 approvals: $\mid \mathcal{V} \backslash N\left[v_{i}\right] \succ b \succ p \succ a \succ N\left[v_{i}\right]$

$k$ voters with 1 approval: $a$

1 voter with $n$ approvals: $\mathcal{V}$

$n+1-k$ voters with $n+3$ approvals: $a \succ b \succ p \succ \mathcal{V} \mid$

The cost of increasing the approval count arbitrarily is 0 in any of the votes in $X=\left\{x_{1}, \ldots, x_{n}\right\}$, and is $+\infty$ in all other votes; our budget is 0 . Observe that in $E$ we have $s_{1}(a)=n+1, s_{2}(b)=n+1-k, s_{3}(p)=n+1-k$, and $s_{n+3}\left(v_{i}\right)=n+2-k$ for each $i$. Thus, no candidate has strict majority (that is, 
$n+2$ points) in any round, so the winner is candidate $a$, who has the largest number of approvals.

Let $\mathbf{t}$ be some minimal push action for $I$. Observe that since we can only increase the approval counts, $s_{1}(a)$ is $n+1$ in $p \operatorname{sh}(E, \mathbf{t})$ as well. Hence, applying $\mathbf{t}$ must increase $p$ 's score by at least $k$ in order for $p$ to beat $a$ in some round. To achieve this, $\mathbf{t}$ must increase the approval count in at least $k$ votes in $X$. On the other hand, suppose that we bribe more than $k$ voters in $X$ to approve $p$. Then $b$ gets at least $n+2$ points in some round of the resulting election; let $j$ be the first such round. As all voters prefer $b$ to $p$, it cannot be the case that $p$ gets $n+2$ points in round $j$, so this bribery is not successful. Hence, applying t must increase $p$ 's approval count by exactly $k$, via bribing a subset of voters $S \subseteq X$ of size $k$. Note that this implies $\alpha(I)=k$ as well. Let $S=\left\{s_{1}, \ldots, s_{k}\right\}$.

Now, looking at the scores of the candidates in $\mathcal{V}$, one can see that a support bribery that bribes voters in $S$ to increase $p$ 's score is successful if and only if each $v_{i} \in \mathcal{V}$ receives at most $k-1$ additional points from the voters in $S$. This holds if and only if each vertex is missing from at least one of the sets $\mathcal{V} \backslash N\left[s_{1}\right], \ldots, \mathcal{V} \backslash N\left[s_{k}\right]$. This is equivalent to $s_{1}, \ldots, s_{k}$ being a dominating set. Thus, the proof is complete.

Since the hardness result of Theorem 5 for Fallback-SUPPORT BRIBERY holds even if all bribery costs are either 0 or $+\infty$, it follows that this problem does not admit an approximation algorithm with a bounded approximation ratio.

Theorem 5 shows that Fallback-SUPPORT BRIBERY is W[2]-hard with respect to the parameter $\alpha$. Given that we have $\beta(I) \geq \alpha(I)$ for each instance $I$, it is natural to ask whether Fallback-SUPPORT BRIBERY remains hard if even $\beta$ is small, i.e., every minimal push action only makes small changes to the approval counts. In Section 5, we will see that even a very restricted version of this problem remains hard. More precisely, in Theorem 7 we will prove that SUPPORT BRIBERY for each of SP-AV, simplified Fallback voting, and Fallback voting remains NPhard and also $\mathrm{W}[1]$-hard with respect to parameter $\beta$, even for single-peaked electorates and unit costs, i.e., when $\sigma^{i}(k)=|k|$ for each $k$ and each $i=1, \ldots, n$.

However, the hardness proof in Theorem 7 (see Section 5) heavily relies on the fact that unit cost functions allow us to increase approval counts in some of the votes while decreasing them in some other votes. In contrast, we will now prove that if all cost functions are positive or all cost functions are negative, (simplified) Fallback-SUPPORT BRIBERY is fixed-parameter tractable with respect to $\beta^{\prime}$ (and hence also with respect to $\beta$ ).

Theorem 6. SUPPORT BRIBERY for SP-AV, simplified Fallback voting, and Fallback voting is $\mathrm{FPT}$ with respect to parameter $\beta^{\prime}$ (the minimum total change in approval counts over all optimal briberies), as long as either all bribery cost functions are positive or all bribery cost functions are negative.

Proof. Suppose we are given an instance $I=(C, V, \Sigma, p)$ of SUPPORT BRIBERY with $|V|=n$. It will be convenient to assume that we also given $\beta^{\prime}=\beta^{\prime}(I)$; if this is not the case, we can try each possible value of $\beta^{\prime}$ in an increasing fashion. We will present an algorithm that works for both the simplified and the 
classic variant of Fallback voting; the algorithm can easily be modified (indeed, simplified) for SP-AV.

Under the Fallback rule, a candidate can win by either (a) having the highest number of approvals in the Bucklin winning round or (b) having the highest number of approvals when there is no Bucklin winning round; for simplified Fallback rule, condition (a) changes to $\left(a^{\prime}\right)$ obtaining a majority of approvals in the Bucklin winning round. To take into account briberies that ensure $p$ 's victory via case (b), we view this case as an "extra round" in which the candidates with the highest number of approvals win. This way we can treat all cases in a uniform manner (it will be clear how to handle minor differences hidden by this notation).

A bribery $\mathbf{t}=\left(t_{1}, \ldots, t_{n}\right)$ with $\sum_{i=1}^{n}\left|t_{i}\right| \leq \beta^{\prime}$ has the following two properties, which will be used by our algorithms:

(1) $\mathbf{t}$ can change the approval scores of at most $\beta^{\prime}$ candidates, and

(2) $\mathbf{t}$ can change the approval score of each candidate by at most $\beta^{\prime}$.

If $\mathbf{t}$ makes $p$ win in round $\ell$ of the election $p \operatorname{sh}(C, V, \mathbf{t})$ while changing its approval score by $\delta(p)$, then we say that $\mathbf{t}$ is an $(\ell, \delta(p))$-bribery; here $\ell$ may also refer to the extra round. As argued above, we can restrict ourselves to $(\ell, \delta(p))$-briberies with $0 \leq \delta(p) \leq \beta^{\prime}$.

Negative cost functions. Suppose first that each cost function is negative; in this case any bribery can only decrease a candidate's score.

We use a bounded search tree approach. By "guessing" an answer to a question, we always mean branching in the search tree according to all the possible ways of answering this question. Our algorithm will branch at most $f\left(\beta^{\prime}\right)=\beta^{\prime}+2$ times, and in each case it will branch into at most $g\left(\beta^{\prime}\right)=3^{\beta^{\prime}+1}$ directions. Moreover, our algorithm will make at most a linear number of steps until reaching a leaf of the search tree. This ensures that its running time can be bounded by $O\left(g(k)^{f(k)}|I|\right)=3^{O\left(\beta^{\prime 2}\right)}|I|$, and hence our problem is fixed-parameter tractable with respect to $\beta^{\prime}$.

We first make some observations regarding our input instance. If $p$ is approved by at most $\lfloor n / 2\rfloor$ voters in $(C, V)$, then its only chance to win in $p \operatorname{sh}(C, V, \mathbf{t})$ is to have the highest number of approvals in the extra round. On the other hand, suppose that $p$ is approved by at least $\lfloor n / 2\rfloor+1$ voters in $(C, V)$. It may then be beneficial to bribe some of the voters who approve $p$ in order to prevent some other candidates from winning in an earlier round. Let $\ell_{0}$ be the earliest round in which $p$ receives $\lfloor n / 2\rfloor+1$ approvals, let $\ell_{1}, \ldots, \ell_{q}$ denote the subsequent rounds where $p$ receives additional approval points, and let $\ell_{q+1}$ denote the extra round; naturally, $\ell_{0}<\ell_{1}<\cdots<\ell_{q} \leq \ell_{q+1}$. Now, suppose that there is a bribery $\mathbf{t}$ that makes $p$ a winner in $p \operatorname{sh}(C, V, \mathbf{t})$. If $\mathbf{t}$ does not decrease the number of approvals that $p$ has, then $p$ wins in round $\ell_{0}$ in $p \operatorname{sh}(C, V, \mathbf{t})$. If $\mathbf{t}$ bribes some of the voters who approve $p, p$ wins in some round $\ell_{q^{\prime}}$ with $q^{\prime} \in\{0,1, \ldots, q, q+1\}$. We will now argue that $q^{\prime} \leq \beta^{\prime}$.

To see why this is the case, suppose that under $\mathbf{t}$ we bribe $x$ voters who approve $p$ and rank her in top $\ell_{0}$ positions and $y$ voters who approve $p$ and rank her in position $\ell_{1}$ or lower; note that $x+y \leq \beta^{\prime}$. Let $L=\left\{\ell_{i_{1}}, \ldots, \ell_{i_{r}}\right\} \subseteq$ 
$\left\{\ell_{1}, \ldots, \ell_{q}\right\}$ be the list of rounds such that for each $\ell \in L$ it holds that $p$ receives one or more approval points in round $\ell$ in $(C, V)$, but not in $p \operatorname{sh}(C, V, \mathbf{t})$ (i.e., in $\mathbf{t}$ all voters who approve $p$ and rank it in position $\ell$ are bribed not to approve $p)$. By construction, we have $|L| \leq y$. Renumber the elements of $\left\{\ell_{1}, \ldots, \ell_{q}\right\} \backslash L$ as $\ell_{j_{1}}, \ldots, \ell_{j_{s}}$. In $p \operatorname{sh}(C, V, \mathbf{t})$ candidate $p$ has at least $\lfloor n / 2\rfloor+1-x$ approvals in round $\ell_{0}$, and it gains at least one approval point in each of the rounds $\ell_{j_{1}}, \ldots, \ell_{j_{s}}$, so it is approved by a strict majority of voters in round $\ell_{j_{x}}$. Since $|L| \leq y$, we have $j_{x} \leq x+y \leq \beta^{\prime}$. Hence, if $p$ is approved by at least $\lfloor n / 2\rfloor+1$ voters in $(C, V)$ and $\mathbf{t}$ is a successful bribery, then $p$ wins in $p \operatorname{sh}(C, V, \mathbf{t})$ in one of the rounds $\ell_{0}, \ldots, \ell_{q^{\prime}}$, where $q^{\prime}=\min \left\{\beta^{\prime}, q+1\right\}$.

We now describe our algorithm. First, the algorithm guesses the round $\ell$ in which $p$ wins in $\operatorname{psh}(C, V, \mathbf{t})$ and the number $\delta(p)$ of approvals that $p$ loses until this round; in other words, we guess $(\ell, \delta(p))$ for which $\mathbf{t}$ is an $(\ell, \delta(p))$-bribery. By our previous observations, there are $\beta^{\prime}+1$ choices of $\ell$ and $\beta^{\prime}+1$ choices of $\delta(p)$.

The algorithm then computes the set of candidates that have to lose at least one point as a result of $\mathbf{t}$ (this computation depends on whether we consider classic or simplified Fallback voting). Let $R$ be the set that consists of these candidates as well as candidate $p$; we say that the candidates in $R$ are relevant. By Observation (1), if $I$ is solvable and we guessed $\ell$ and $\delta(p)$ correctly, the set $R$ contains at most $\beta^{\prime}+1$ candidates. The algorithm also computes integers $\delta_{\ell-1}(c)$ and $\delta_{\ell}(c)$ for each $c \in R \backslash\{p\}$ such that:

(3) an $(\ell, \delta(p))$-bribery makes $p$ a winner if and only if each candidate $c \in R \backslash\{p\}$ loses at least $\delta_{\ell-1}(c)$ points until round $\ell-1$, and at least $\delta_{\ell}(c)$ points until round $\ell$.

The procedure for computing these integers depends on whether we consider classic or simplified Fallback voting. In particular, we always have $\delta_{\ell-1}(c) \leq \delta_{\ell}(c)$ and under simplified Fallback voting we have $\delta_{\ell-1}(c)=\delta_{\ell}(c)$. We set $\delta_{\ell-1}(c)=0$ if $\ell=1$.

Next, the algorithm partitions the set $\left\{(v, t) \mid v \in V, 1 \leq t \leq \beta^{\prime}\right\}$ into equivalence classes. By applying a pair $\left(v^{i}, t\right)$ we mean bribing voter $v^{i}$ to decrease her approval count from $\ell^{i}$ to $\ell^{i}-t$. We say that $(v, t)$ and $\left(v^{\prime}, t^{\prime}\right)$ are equivalent if for each $c_{r} \in R$ it holds that applying $(v, t)$ has the same effect on $c_{r}$ as applying $\left(v^{\prime}, t^{\prime}\right)$. More formally, $(v, t)$ and $\left(v^{\prime}, t^{\prime}\right)$ are equivalent if for each $c_{r} \in R$ it holds that (i) $(v, t)$ and $\left(v^{\prime}, t^{\prime}\right)$ decrease the number of approvals $c_{r}$ gets until round $\ell-1$ by the same amount and (ii) $(v, t)$ and $\left(v^{\prime}, t^{\prime}\right)$ decrease the number of approvals $c_{r}$ gets until round $\ell$ by the same amount. A pair $(v, t)$ can behave in three possible ways with respect to $c_{r}$ : it can leave its approval count until round $\ell$ (and hence also its approval count until round $\ell-1$ ) unchanged, it can decrease by one its approval count in round $\ell$ (but not in earlier rounds), or it can decrease by one its approval count until round $\ell-1$, thereby also decreasing its approval count until round $\ell$. Therefore, there are at most $3^{|R|} \leq 3^{\beta^{\prime}+1}$ equivalence classes. Note that applying some pair $(v, t)$ instead of another pair that is equivalent to it does not change whether a given push action is successful or not. 
Finally, the algorithm proceeds as follows: it guesses an equivalence class, picks a cheapest pair $\left(v^{i}, t\right)$ from this class that has not been applied so far (breaking ties arbitrarily), and applies it. By construction, in some of the at most $3^{|R|}$ branches the algorithm will choose a pair that can be extended to an optimal bribery, if there exists one. It repeats this step until it reaches the bound $\beta^{\prime}$ on the total approval count modification; this means at most $\beta^{\prime}$ branchings. By the arguments above, a minimum-cost solution for $I$ can be obtained by taking a minimum-cost bribery among all the successful briberies (that is, ones that ensure $p$ 's victory) considered.

Positive cost functions. Let us now focus on the case of positive cost functions, where each bribery can only increase a candidate's number of approvals.

For each possible $\ell$ and $\delta(p)$, the algorithm tries to find a minimal $(\ell, \delta(p))$ bribery $\mathbf{t}$. This means considering at most $|C|+1$ possibilities for $\ell$, and, by (2), at most $\beta^{\prime}+1$ possibilities for $\delta(p)$. Note that under positive cost functions a minimal bribery always bribes voters so that in each modified vote the approval count is equal to the rank of $p$. Consequently, the number of bribed voters in a minimal $(\ell, \delta(p))$-bribery is $\delta(p)$, and no other candidate receives additional approvals in round $\ell$.

Having picked $\ell$ and $\delta(p)$, the algorithm tries all possible ways of choosing a (multi)set of positive integers $\{t[1], \ldots, t[\delta(p)]\}$ with $t[1]+\cdots+t[\delta(p)]=\beta^{\prime}$, corresponding to the increase of the approval counts of the bribed voters. In other words, it guesses the non-zero elements of $\mathbf{t}$ (but not their positions in $\mathbf{t}$ ). There are at most $\beta^{\prime \delta(p)} \leq \beta^{\prime \beta^{\prime}}$ possibilities.

Then, the algorithm computes integers $\delta(c), c \in C \backslash\{p\}$, such that

(3') an $(\ell, \delta(p))$-bribery makes $p$ a winner if and only if each candidate $c \in C \backslash\{p\}$ gains at most $\delta(c)$ points until round $\ell-1$ (and hence until round $\ell$ as well), assuming that $p$ does not get a majority of votes in round $\ell-1$ or earlier and gains exactly $\delta(p)$ points until round $\ell$.

The procedure for computing $\delta(c)$ depends on whether we consider simplified or classic Fallback voting, and is polynomial-time implementable in either case.

The next step of the algorithm uses the color-coding technique of Alon, Yuster, and Zwick [1]. This results in a randomized algorithm with one-sided error, which produces a correct output with probability at least $2^{-\delta(p) \beta^{\prime}}$; this algorithm can then be derandomized using standard methods.

We associate a color with each bribed voter. Colors are denoted by integers between 1 and $\delta(p)$; recall that $\mathbf{t}$ bribes exactly $\delta(p)$ voters, and we have $\delta(p) \leq$ $\beta^{\prime}$. We construct a coloring $A: C \rightarrow 2^{\{1, \ldots, \delta(p)\}}$ by assigning each candidate $c \in \bar{C}$ a random subset $A(c)$ of colors chosen uniformly and independently. Intuitively, for a candidate $c \in C \backslash\{p\}$ that obtains additional approvals as a result of the bribery $\mathbf{t}$, the set $A(c)$ corresponds to the set of bribed voters in $p \operatorname{sh}(C, V, \mathbf{t})$ who grant an additional approval to $c$. For candidate $p$, the set $A(p)$ corresponds to the set of bribed voters that grant an additional approval to $p$ until round $\ell-1$. We say that $A$ is valid for a candidate $c \in C \backslash\{p\}$ if $|A(c)| \leq \delta(c)$; it is valid for $p$ if $|A(p)| \leq\lfloor n / 2\rfloor-s_{\ell-1}^{\prime}(p)$, where $s_{\ell-1}^{\prime}(p)$ denotes the number of approval 
points $p$ gets in the original election until round $\ell-1$. A coloring is valid if it is valid for all candidates in $C$. The concept of validity reflects the fact that we have to fulfill condition (3').

Given a valid coloring of the candidates $A$, the algorithm computes the set of admissible colors for each voter $v^{i} \in V$. A color $x \in\{1, \ldots, \delta(p)\}$ is admissible for $v^{i}$ if the following holds:

(a) $\operatorname{rank}\left(p, v^{i}\right)=\ell^{i}+t[x]$ and $t[x] \leq \ell-\ell^{i}$, i.e., in order to give an extra approval to $p$ in $v^{i}$ (in round $\ell$ or earlier), the approval count has to be increased by exactly $t[x]$

(b) if $\operatorname{rank}\left(p, v^{i}\right)<\ell$, then $x \in A(p)$;

(c) for each candidate $c$ with $\ell^{i}<\operatorname{rank}\left(c, v^{i}\right)<\operatorname{rank}\left(p, v^{i}\right)$, we have $x \in A(c)$.

We say that a collection $v^{i_{1}}, \ldots, v^{i_{\delta(p)}}$ of voters is proper if for each $x, 1 \leq x \leq$ $\delta(p)$, the color $x$ is admissible for voter $v^{i_{x}}$.

Finally, the algorithm computes a proper collection of voters $v^{i_{1}}, \ldots, v^{i_{\delta(p)}}$ that minimizes the cost of a bribery where we bribe each voter $v^{i_{x}}$ to increase his approval count by $t[x]$. To do this, it finds a minimum-weight maximal matching in the bipartite graph where we have the set of voters who do not approve $p$ on one side, $\delta(p)$ colors on the other side, there is an edge from each voter to all colors that are admissible for him, and the weight of each edge corresponds to the cost of bribing the respective voter to shift his approval threshold so as to approve $p$. Note that a matching of size $\delta(p)$ in this graph corresponds to a proper collection of voters; if there is no such matching in the graph, then the algorithm does not output anything.

The correctness of our algorithm is based on the following key observation: if a collection of voters $v^{i_{1}}, \ldots, v^{i_{\delta(p)}}$ is proper, then increasing the approval count of $v^{i_{x}}$ by $t[x]$ for each $x=1, \ldots, \delta(p)$ makes $p$ a winner in round $\ell$. To see this, note that condition ( $\left.3^{\prime}\right)$ is satisfied if we apply such a bribery. Indeed, condition (a) ensures that $p$ gains the necessary $\delta(p)$ points until round $\ell$, condition (b) together with the definition of validity for $p$ ensures that $p$ does not win before round $\ell$, and condition (c) and the definition of validity for candidates in $C \backslash\{p\}$ that are affected by the bribery ensures that no candidate gains more extra approvals than it is allowed to.

To complete the proof of correctness, it remains to show that if there exists an $(\ell, \delta(p))$-bribery $\mathbf{t}$ of cost at most $B$ that increases the approval counts of voters $v^{i_{1}}, \ldots, v^{i_{\delta(p)}}$ by $t[1], \ldots, t[\delta(p)]$, then our algorithm outputs a bribery of cost at most $B$ with probability at least $2^{-\delta(p) \beta^{\prime}}$. To see this, consider the event that the candidates affected by $\mathbf{t}$ are colored "as expected", meaning that each candidate whose additional approvals under $\mathbf{t}$ come from voters in the set $\left\{v^{i_{x}} \mid x \in X\right\}$ for some $X \subseteq\{1, \ldots, \delta(p)\}$ receives the set $X$ of colors during the coloring process. For each such candidate this holds with probability $2^{-\delta(p)}$. Since there are at most $\beta^{\prime}$ candidates that receive additional approvals as a result of the bribery $\mathbf{t}$, it follows that with probability at least $2^{-\delta(p) \beta^{\prime}}$ it holds that for each $x$, the color $x$ will be admissible for voter $v^{i_{x}}$. Whenever this holds, the algorithm will consider $\mathbf{t}$ when searching for a cheapest proper collection of voters, and hence the output will be a (successful) bribery of cost at most $B$. 
Consequently, the algorithm indeed produces a correct output with probability at least $2^{-\delta(p) \beta^{\prime}} \geq 2^{-\beta^{\prime 2}}$.

Let us now analyze the running time of our algorithm. After choosing $\ell$, $\delta(p)$, and the integers $t[1], \ldots, t[\delta(p)]$, the coloring process and the computation of admissible colors for each of the voters can be implemented in linear time. A minimum-weight matching can be identified in polynomial time by, e.g., the Hungarian method [35]. The branchings in the beginning of the algorithm contribute a factor of $\beta^{\prime \beta^{\prime}}|C|$ to the running time, yielding an overall running time of $O\left(\beta^{\prime \beta^{\prime}}|I|^{O(1)}\right)$.

To derandomize the algorithm, one can use $\left(\beta^{\prime}|C|, \beta^{\prime 2}\right)$-universal sets [39]; the resulting algorithm is still in FPT with respect to the parameter $\beta^{\prime}$.

\section{$5 \quad$ Support Bribery for Single-Peaked Electorates}

One possible way to circumvent the hardness results of Section 4 is to study the complexity of SUPPORT BRIBERY under restricted preferences. Recent work [5, $8,11,29]$ shows that many hard problems in computational social choice become easy if the voters' preferences can be assumed to be single-peaked. In the next theorem we show that this is not the case for SUPPORT BRIBERY, as this problem remains NP-hard (and also W[1]-hard with parameter $\beta$ ) even for single-peaked electorates, for each of the voting rules considered in this paper.

Theorem 7. SUPPORT BRIBERY under single-peaked preferences is NP-hard and $\mathrm{W}[1]$-hard with respect to parameter $\beta$ for each of $S P-A V$, Fallback and simplified Fallback, even if $\sigma^{i}(k)=|k|$ for each $k$ and each $i=1, \ldots, n$.

Proof. We present a parameterized reduction from the W[1]-hard MULTICOLORED CLIQUE problem [32]; the same reduction works for SP-AV, Fallback, and simplified Fallback. Consider an instance of MULTICOLORED CLIQUE given by an integer $k$ and a graph $G=(\mathcal{V}, \mathcal{E})$ with the vertex set $\mathcal{V}=\left\{\nu_{1}, \ldots, \nu_{N}\right\}$ partitioned into $k$ independent sets $\mathcal{V}^{1}, \ldots, \mathcal{V}^{k}$. Without loss of generality we assume that $\nu_{1} \in \mathcal{V}^{1}$ and $\nu_{N} \in \mathcal{V}^{k}$.

We will construct an instance $I$ of support bribery with unit costs for a single-peaked election. We will set the budget $B=2 k^{3}-k$ and ensure that an optimal bribery has cost $B$ if and only if $G$ contains a $k$-clique, and that there always is a successful bribery of cost at most $B+1$. Since $I$ has unit costs, this would imply $B \leq \beta(I) \leq \beta^{\prime}(I) \leq B+1$.

We form an election $(C, V)$ contained in $I$ as follows. For each $i=1, \ldots, k$ and each vertex $\nu_{a} \in \mathcal{V}^{i}$, we introduce a candidate set $C\left(\nu_{a}\right)=\left\{c_{a}^{j} \mid 1 \leq j \leq\right.$ $k, j \neq i\}$, and we set $C_{\mathcal{V}}=\bigcup_{\nu \in \mathcal{V}} C(\nu)$. We then set $C=C_{\mathcal{V}} \cup\{p, q\} \cup D$, where $p$ is our preferred candidate and $D$ is a set of dummies (see the next paragraph).

We define a linear order $\triangleleft$ on the set of candidates as follows. The first candidate in this order is $q$, the last one is $p$, and candidate $c_{a}^{i}$ precedes $c_{b}^{j}$ if either $a<b$, or $a=b$ but $i<j$. The dummy candidates are placed between candidates

that are adjacent in the sequence $q, c_{1}^{2}, c_{1}^{3}, \ldots, c_{N}^{k-2}, c_{N}^{k-1}, p$. Specifically, we place $2 B$ dummies between $q$ and $c_{1}^{2}$, as well as between $c_{N}^{k-1}$ and $p$, and we place two 
dummies between every pair of adjacent candidates in $C_{\mathcal{V}}$. The linear order $\triangleleft$ is illustrated below ( $\diamond$ signs stand for the dummies).

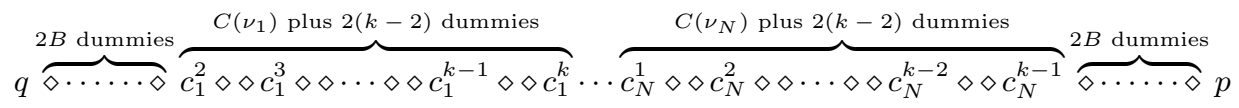

For each vertex $\nu_{a} \in \mathcal{V}$, we introduce a voter $w_{a}$, and for each edge $\left\{\nu_{a}, \nu_{b}\right\} \in$ $\mathcal{E}$, we introduce a voter $w_{\{a, b\}}$. We let $W_{\mathcal{V}}=\left\{w_{a} \mid \nu_{a} \in \mathcal{V}\right\}$ and $W_{\mathcal{E}}=\left\{w_{\{a, b\}} \mid\right.$ $\left.\left\{\nu_{a}, \nu_{b}\right\} \in \mathcal{E}\right\}$. To define the preferences of these voters, we need additional notation. For each candidate $c$ and each integer $i$, we denote by $\operatorname{prec}^{i}(c)$ the $i$-th candidate before $c$ in $\triangleleft$, and we denote by $\operatorname{succ}^{i}(c)$ the $i$-th candidate after $c$ in $\triangleleft$. We sometimes write $\operatorname{succ}(c)$ instead of $\operatorname{succ}^{1}(c)$ and $\operatorname{prec}(c)$ instead of $\operatorname{prec}^{1}(c)$. If $c$ precedes $c^{\prime}$ in $\triangleleft$, we denote by $c \cdots c^{\prime}$ the sequence of candidates from $c$ to $c^{\prime}$ (inclusively) with respect to $\triangleleft$; the same sequence in reverse is denoted by $c^{\prime} \cdots c$. The preference orders and approval counts of voters $w_{a}$ and $w_{\{a, b\}}$, where $\nu_{a} \in \mathcal{V}^{i}, \nu_{b} \in \mathcal{V}^{j},\left(\nu_{a}, \nu_{b}\right) \in \mathcal{E}$, and $a<b$, are given below (to simplify notation, we assume $i, j \notin\{1, k\}$; it is easy to modify the construction for the case $i=1$ or $j=k$ ). In what follows, the order of the non-approved candidates not shown in the preference lists can be defined in any way that results in preferences that are single-peaked with respect to $\triangleleft$.

$$
\begin{array}{ll}
w_{a}: & \operatorname{succ}\left(c_{a}^{k}\right) \cdots \operatorname{prec}^{2 k^{2}-5 k+6}(p) \mid c_{a}^{k} \cdots c_{a}^{1} \succ \operatorname{prec}^{2 k^{2}-5 k+5}(p) \cdots p \\
w_{\{a, b\}}: & \operatorname{succ}\left(c_{a}^{j}\right) \cdots \operatorname{prec}\left(c_{b}^{i}\right) \succ c_{a}^{j} \succ c_{b}^{i} \succ \operatorname{prec}\left(c_{a}^{j}\right) \succ \operatorname{succ}\left(c_{b}^{i}\right) \mid
\end{array}
$$

We will now add extra votes to ensure that each candidate $c \in C_{\mathcal{V}} \cup\{q\}$ receives $L$ approvals, each dummy receives fewer than $L$ approvals, while $p$ receives $L-k$ approvals in $(C, V)$ for some sufficiently large integer $L$.

To do so, we first make sure that all candidates in $C_{\mathcal{V}}$ have the same number of approvals. To this end, if some candidate $c \in C_{\mathcal{V}}$ has fewer approvals than another candidate in $C_{\mathcal{V}}$, we add a vote $c \cdots \operatorname{prec}^{B}\left(c_{1}^{2}\right) \mid$ and a vote $c \cdots \operatorname{succ}^{B}\left(c_{N}^{k-1}\right) \mid$ to $V$. These two votes provide one extra approval point to each candidate in $C_{\mathcal{V}} \backslash\{c\}$ and two extra approval points to $c$. Thus, by adding such pairs of votes iteratively, we can ensure that each candidate in $C_{\mathcal{V}}$ has the same score. After equalizing the scores of all candidates in $C_{\mathcal{V}}$ in this manner, we introduce $|\mathcal{V}|+|\mathcal{E}|+B+1$ additional pairs of such votes for each $c \in C_{\mathcal{V}}$; this ensures that at this point each dummy receives strictly fewer approvals than candidates in $C_{\mathcal{V}}$. Let the resulting score of the candidates in $C_{\mathcal{V}}$ be $L$; note that $L>2 B+k$. To obtain the required scores for $p$ and $q$, we add $L-k$ voters approving $p$ only and $L$ voters with preferences of the form $q \cdots \operatorname{succ}^{B}(q) \mid$. We denote by $V_{\text {init }}$ the set of voters added to adjust the initial scores; we let $V=W_{\mathcal{V}} \cup W_{\mathcal{E}} \cup V_{\text {init }}$.

This completes the construction, which is clearly polynomial in size. Note that the total approval score of each candidate is as required. Also, it is straightforward to check that the preferences of all voters are single-peaked with respect to the linear order $\triangleleft$.

It remains to show the correctness of the reduction. Note that the total number of voters in $V$ is at least $3 L-k$, while every candidate has at most 
$L$ approvals. As $L>k$, it follows that no candidate is approved by a strict majority of voters in $(C, V)$. Moreover, since $L>2 B+k=4 k^{3}-k$, after any bribery that does not exceed the budget the score of each candidate is at most $L+B<(3 L-k) / 2<\lfloor|V| / 2\rfloor+1$, and hence no candidate is approved by a majority of voters after any such bribery. Thus $p$ can be made a winner under each of SP-AV, Fallback, and simplified Fallback if and only if $p$ obtains the maximum number of approvals after some bribery. Thus, from now on, when we speak of a score of a candidate or a candidate's number of points, we refer to this candidate's number of approvals. To follow our arguments, the reader may find it useful to keep Figure 2 in mind.

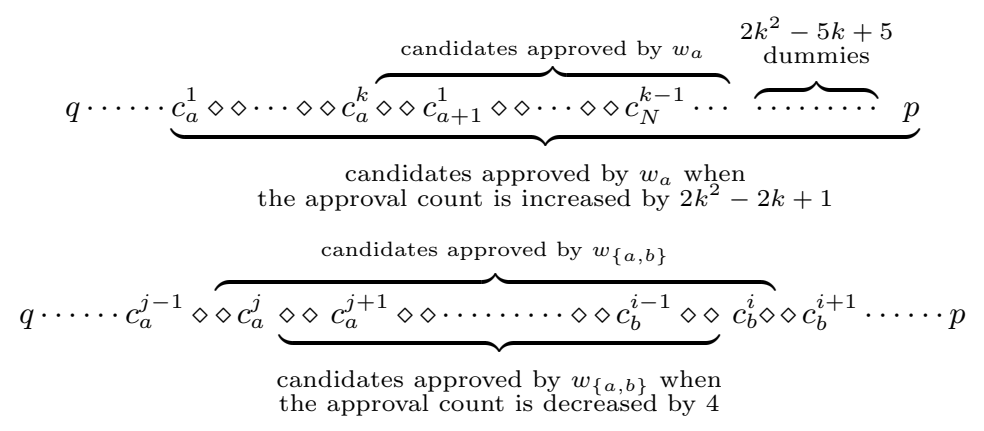

Fig. 2. Preferences of voters $w_{a}$ and $w_{\{a, b\}}$ with respect to $\triangleleft$.

First, suppose that there is a minimal support bribery $\mathbf{t}$ of cost at most $B$. Observe that lowering the approval counts in any of the votes $q \cdots \operatorname{succ}^{B}(q) \mid$ in order to decrease $q$ 's score would have a cost of $B+1$. Thus, $\mathbf{t}$ cannot decrease $q$ 's score, and hence it must increase $p$ 's score by at least $k$ points. Since $p$ is preceded by $B$ dummies in $\triangleleft$, it follows that in $\mathbf{t}$ the bribed voters form a subset of $W_{\mathcal{V}}$.

Note that increasing the approval count of a voter $w_{a} \in W_{\mathcal{V}}$ so that $p$ obtains an additional point has cost $(3 k-5)+\left(2 k^{2}-5 k+5\right)+1=2 k^{2}-2 k+1$. Since $(k+1)\left(2 k^{2}-2 k+1\right)=2 k^{3}-k+1>B$, at most $k$ voters can be bribed in this way without exceeding the budget. Note also that bribing $k+1$ voters from $W_{\mathcal{V}}$ in this manner would make $p$ a winner at the cost of $2 k^{3}-k+1=B+1$, which shows that $\beta(I) \leq B+1$.

Since $p$ 's score needs to be increased by at least $k$, we can conclude that $\mathbf{t}$ must bribe exactly $k$ voters from the set $W_{\mathcal{V}}$, while increasing $p$ 's score by exactly $k$. Let $w_{s_{1}}, w_{s_{2}}, \ldots, w_{s_{k}}$ denote these bribed voters. We are going to show that the vertex set $S=\left\{\nu_{s_{1}}, \ldots, \nu_{s_{k}}\right\}$ is a solution of the MULTiCOLORED CLIQUE instance.

Observe that when voters $w_{s_{1}}, w_{s_{2}}, \ldots, w_{s_{k}}$ are bribed, each of the $k(k-1)$ candidates in $C^{*}=\bigcup_{i=1}^{k} C\left(\nu_{s_{i}}\right)$ receives one additional point. Since each of these candidates has $L$ approvals in the original election $(C, V)$, and the final score of 
$p$ in $p \operatorname{sh}(C, V, \mathbf{t})$ is $L, \mathbf{t}$ must bribe some additional voters to lower their approval count so that each candidate in $C^{*}$ loses at least one point. Bribing a voter in $V_{\text {init }} \cup W_{\mathcal{V}}$ to decrease the score of any candidate in $C_{\mathcal{V}}$ costs more than $B$. Thus, to prevent the candidates in $C^{*}$ from beating $p$, t must bribe some voters in $W_{\mathcal{E}}$; let $W_{\mathcal{E}}^{*}$ denote the set of these voters.

Bribing a voter $w_{\{a, b\}} \in W_{\mathcal{E}}^{*}$ may:

(i) decrease the score of exactly one candidate in $C_{\mathcal{V}}$ at a cost of 3 , or

(ii) decrease the score of exactly two candidates in $C_{\mathcal{V}}$ at a cost of 4 , or

(iii) decrease the score of $\ell$ candidates in $C_{\mathcal{V}}$ for some $\ell \geq 3$ at a cost of $3(\ell-2)+4$.

Thus, decreasing the score of any candidate in $C^{*}$ has a cost of at least 2 per candidate; moreover, equality can only be achieved if case (ii) holds for each of the bribed voters. Hence, in order to decrease the approval score by one for each of the $k(k-1)$ candidates in $C^{*}$, the briber needs to spend at least $2 k(k-1)$. We have argued that the briber has to spend $k\left(2 k^{2}-2 k+1\right)$ on bribing voters in $W_{\mathcal{V}}$. Thus, her remaining budget is $B-k\left(2 k^{2}-2 k+1\right)=2 k(k-1)$, i.e., $\mathbf{t}$ must bribe exactly $\left(\begin{array}{l}k \\ 2\end{array}\right)$ voters from $W_{\mathcal{E}}$, lowering the approval counts of each voter in $W_{\mathcal{E}}^{*}$ by exactly 4 ; moreover, both non-dummy candidates who lose points as a result of this bribery should be members of $C^{*}$.

Now, fix some vertex $\nu_{x} \in S$, and let $i$ be the index for which $\nu_{x} \in \mathcal{V}^{i}$. By the definition of $C^{*}$ and $S$, we have $C\left(\nu_{x}\right) \subseteq C^{*}$. Therefore, for each $j$ with $1 \leq j \leq k, j \neq i$, the candidate $c_{x}^{j} \in C^{*}$ must be among the last four approved candidates of some voter in $W_{\mathcal{E}}^{*}$. By construction of $W_{\mathcal{E}}$, this voter must be $w_{\{x, y\}}$ for some $y$ where $\left\{\nu_{x}, \nu_{y}\right\} \in \mathcal{E}$ and $\nu_{y} \in \mathcal{V}^{j}$. As argued in the previous paragraph, this means that $c_{y}^{i} \in C^{*}$ and hence $\nu_{y} \in S$. Thus, for every vertex $\nu_{x}$ in the set $S$, we can conclude that each class $\mathcal{V}^{j}$ with $\nu_{x} \notin \mathcal{V}^{j}$ contains a vertex in $S \cap \mathcal{V}^{j}$ that is adjacent to $\nu_{x}$. As this holds for each $\nu_{x} \in S$, it follows that $S$ forms a clique of size $k$ in $G$.

For the converse direction, suppose that vertices $\nu_{s_{1}}, \ldots, \nu_{s_{k}}$ form a clique of size $k$ in $G$. It can be easily verified that lowering the approval counts of each of the voters $w_{\left\{s_{i}, s_{j}\right\}}$ with $1 \leq i<j \leq k$ by 4 and increasing the approval counts of each of the voters $w_{s_{i}}$ with $1 \leq i \leq k$ by $2 k^{2}-2 k+1$ results in a successful bribery of cost $4\left(\begin{array}{l}k \\ 2\end{array}\right)+\left(2 k^{2}-2 k+1\right) k=B$.

In Theorem 7 we proved that, even for single-peaked preferences and unit costs functions, SP-AV-SUPPORT BRIBERY does not admit an FPT algorithm with respect to parameter $\beta$ unless FPT $=\mathrm{W}[1]$. Naturally, this hardness result also holds for the smaller parameter $\beta^{\prime}$. In contrast, we will now describe an algorithm that is FPT with respect to parameter $\beta^{\prime}$ and for any fixed $\varepsilon>0$ outputs an $(1+\varepsilon)$-approximation for this variant of SUPPORT BRIBERY.

Theorem 8. For any fixed $\varepsilon>0$, SP-AV-SUPPORT BRIBERY for single-peaked preferences can be $(1+\varepsilon)$-approximated by an algorithm that is FPT with respect to $\beta^{\prime}$, as long as $\sigma^{i}(k) \geq 1$ for each $k$ and each $i=1, \ldots, n$.

Proof. Fix a positive constant $\varepsilon$. Let $I=(C, V, \Sigma, p)$ be our input instance of SP-AV-SUPPORT BRIBERY with parameter $\beta^{\prime}$. Just as in the proof of Theorem 6 , 
we can assume that the parameter $\beta^{\prime}$ is given as part of the input; otherwise, we can simply run our algorithm with increasing values of the parameter starting from 1. Set $n=|V|$. For each $i=1, \ldots, n$, we let $v^{i}$ and $\ell^{i}$ denote the $i$-th voter in $V$ and her approval count. Suppose that all voters' preferences are single-peaked with respect to a linear order $\triangleleft$. Let $B$ be our bribery budget.

Let $\mathbf{t}=\left(t_{1}, \ldots, t_{n}\right)$ be some minimal bribery such that $\beta^{\prime}=\sum_{i=1}^{n}\left|t_{i}\right|$. Also, let $V^{+}=\left\{v^{i} \in V \mid t_{i}>0\right\}$ and $V^{-}=\left\{v^{i} \in V \mid t_{i}<0\right\}$.

Since $\mathbf{t}$ is a minimal bribery, each voter $v^{i} \in V^{+} \operatorname{ranks} p$ in position $\ell^{i}+t_{i}$. Thus, given a voter $v^{i}$ who does not approve of $p$, we will refer to the act of increasing $v^{i}$,s approval count by $\operatorname{rank}\left(p, v^{i}\right)-\ell^{i}$ as buying $v^{i}$. The price of $v^{i}$ is the cost of buying her.

Note that since the election is single-peaked, for any voter $v^{i} \in V$ it holds that the set of candidates that $v^{i}$ ranks in top $\ell^{i}$ positions is a contiguous interval of the order $\triangleleft$. Also, the set of candidates that $v^{i}$ ranks in top $\ell^{i}+t_{i}$ positions is a (larger) contiguous interval of $\triangleleft$. Hence, the set of candidates that $v^{i}$ ranks in positions $\ell^{i}+1, \ldots, \ell^{i}+t_{i}$ consists of two intervals of $\triangleleft$; one of them has $p$ as its endpoint and the other may be empty. We write $B\left(v^{i}\right)$ and $S\left(v^{i}\right)$ to denote the two sets of candidates associated with these two intervals, where $B\left(v^{i}\right)$ is the one containing $p$; we refer to $B\left(v^{i}\right)$ and $S\left(v^{i}\right)$ as the base and the shadow of $v^{i}$.

Guessing expensive voters in $V^{+}$. Our algorithm starts by guessing the set of voters $V_{1}^{+} \subseteq V^{+}$whose price is at least $\varepsilon B$. Since the price of each voter is at least 1 , we have $\left|V_{1}^{+}\right| \leq 1 / \varepsilon$. Therefore, the algorithm has to try at most $n^{\lfloor 1 / \varepsilon\rfloor}$ possibilities; for constant $\varepsilon$, this quantity is polynomial in the input size. (Note also that $\left|V_{1}^{+}\right| \leq\left|V^{+}\right| \leq \beta^{\prime}$, so the number of possibilities to be considered at this stage can be bounded as $n^{\min \left\{\lfloor 1 / \varepsilon\rfloor, \beta^{\prime}\right\}}$ ).

Guessing structural properties of $\mathbf{t}$. Next, the algorithm guesses the following information about $\mathbf{t}$ :

1. the total score $s^{*}$ with which $p$ wins in $p \operatorname{sh}(C, V, \mathbf{t})$;

2. the size $k$ of the set $W=V^{+} \backslash V_{1}^{+}$(we will refer to the voters in $W$ as $\left.w_{1}, \ldots, w_{k}\right)$

3. the base $B\left(w_{i}\right)$ for each voter $w_{i} \in W$ (note that the algorithm does not try to guess the set $W$ itself);

4. the size $\left|S\left(w_{i}\right)\right|$ of the shadow for each voter $w_{i} \in W$.

Since we have $0 \leq k \leq \beta^{\prime}$, there are at most $\beta^{\prime}+1$ possible values of $k$. There are also at most $2 \beta^{\prime}+1$ possible choices for $s^{*}$ and at most $\beta^{\prime}+1$ possible choices for the size of each shadow. The base of each voter $w_{i} \in W$ is a set of candidates that is represented by a contiguous interval of $\triangleleft$ of length at most $\beta^{\prime}$ whose left or right endpoint is $p$. This yields $2 \beta^{\prime}-1$ possible choices for each $B\left(w_{i}\right)$. Thus, the total number of possible choices in this guessing step does not exceed $\left(2 \beta^{\prime}+1\right)\left(\beta^{\prime}+1\right)^{\beta^{\prime}+1}\left(2 \beta^{\prime}-1\right)^{\beta^{\prime}}$.

Color-coding step. We will now use color-coding [1]; while this results in a randomized algorithm, we will later explain how to derandomize it. Specifically, we 
associate a color $i$ with each voter $w_{i} \in W$. Given a voter $v$ who does not approve $p$, we say that color $i$ is suitable for $v$ if it holds that $B(v)=B\left(w_{i}\right)$ and $|S(v)|=\left|S\left(w_{i}\right)\right|$. The color-coding step of the algorithm assigns colors to some of the voters in $V$ as follows: for each voter $v \in V \backslash V_{1}^{+}$that does not approve $p$, it chooses uniformly between coloring $v$ with one of the colors suitable for him and leaving $v$ uncolored. Recall that the algorithm does not know the voters $w_{1}, \ldots, w_{k}$, but it has already guessed their bases and the sizes of their shadows, which suffices to compute the set of suitable colors for each voter.

We say that the coloring is successful for a voter $w_{i} \in W$ if $w_{i}$ is colored with his own color $i$; it is successful for a voter $v \in V^{-}$if it leaves him uncolored. A coloring is successful if it is successful for all voters in $W \cup V^{-}$. For each of the voters in $W \cup V^{-}$, the probability that our coloring is successful for him is at least $1 /(k+1)$, so with probability at least $(k+1)^{-\beta^{\prime}}$ the color-coding process results in a successful coloring. Note also that if $k=\beta^{\prime}$ then $V^{-}=\emptyset$, and we can simply color each voter with a suitable color (without leaving voters uncolored) and obtain a successful coloring with probability at least $\beta^{\prime-\beta^{\prime}}$. From now on, we assume that we are given a successful coloring.

Guessing additional voters in $V^{+}$. Observe that if two voters, $v$ and $v^{\prime}$, have the same base and the same shadow, then buying either of these voters has exactly the same effect on each candidate. Hence, when looking for an optimal bribery, we should choose among such voters based on their price. Based on this observation, for each color $i, i=1, \ldots, k$, we will define a set $R_{i}$ of relevant voters, using the following procedure. Let $r=\beta^{\prime 2}+1$. We start with $R_{i}=\emptyset$, consider the voters with color $i$ in order of non-decreasing prices, and place a voter $v$ into $R_{i}$ if and only if no other voter in $R_{i}$ has the same shadow as $v$. We stop when $\left|R_{i}\right|=r$ or when we have considered all voters with color $i$. Let $p_{i}$ denote the maximum price of a voter in $R_{i}$. By construction, we can assume without loss of generality that $w_{i} \in R_{i}$ or the price of $w_{i}$ is at least $p_{i}$ (in which case $\left|R_{i}\right|=r$ ).

Now, our algorithm makes some further guesses. For each color $i, i=1, \ldots, k$, it guesses whether $w_{i} \in R_{i}$; if its guess is "yes", it also guesses which voter in $R_{i}$ is $w_{i}$. For each color such a guess can have at most $r+1$ outcomes, so the algorithm has to try at most $(r+1)^{k} \leq\left(\beta^{\prime 2}+1\right)^{\beta^{\prime}}$ possibilities at this step. Let $V_{2}^{+}$be the set of voters guessed at this step. Set $M=\left\{i \mid w_{i} \notin R_{i}\right\}$; we refer to colors in $M$ as missing colors. Note that for each $i \in M$ we have $\left|R_{i}\right|=r$; this observation will prove useful in our analysis.

Dynamic programming step. Next, the algorithm performs the following calculation for each candidate $c \in C \backslash\{p\}$. It computes the score that $c$ would obtain if we were to buy all voters in $V_{1}^{+}$and $V_{2}^{+}$. It then adds one extra point for each candidate in $B\left(w_{i}\right)$ for each missing color $i$. We denote the resulting quantity by $s^{*}(c)$. Observe that, if we were to buy all voters in $V^{+}$, then the score of $c$ would be at least $s^{*}(c)$. Let $C^{*}=\left\{c \in C \mid s^{*}(c)>s^{*}\right\}$. Note that bribery t buys all voters in $V^{+}$and therefore it has to decrease the score of each candidate $c \in C^{*}$ by at least $s^{*}(c)-s^{*}$ in order to prevent these candidates from beating 
p. Clearly, t achieves this through decreasing the approval counts of the voters in $V^{-}$.

Instead of trying to find the set $V^{-}$, our algorithm simply computes a minimum-cost bribery $\mathbf{t}^{*}$ that decreases the score of each $c \in C^{*}$ by at least $s^{*}(c)-s^{*}$ while bribing only uncolored votes. As we assume that we have a successful coloring, bribing each voter in $V^{-}$according to $\mathbf{t}$ constitutes a feasible solution to this problem, and therefore the cost $\Sigma\left(\mathbf{t}^{*}\right)$ does not exceed the cost of changing the approval count by $t_{i}$ in each vote $v^{i} \in V^{-}$.

The algorithm computes $\mathbf{t}^{*}$ by dynamic programming. We fix an ordering on $C^{*}$ and on the set of uncolored voters. Suppose we have $U$ uncolored voters; clearly, $U \leq n$. Let $m^{*}=\left|C^{*}\right|$. Then for $j \in\{1, \ldots, U\}$ and $s_{1}, \ldots, s_{m^{*}} \in$ $\left\{0, \ldots, \beta^{\prime}\right\}$, we define $f\left(j, s_{1}, \ldots, s_{m^{*}}\right)$ to be the minimum cost of a bribery that bribes a subset of the first $j$ uncolored voters and for each $i=1, \ldots, m^{*}$ decreases the number of approvals of the $i$-th candidate in $C^{*}$ by at least $s_{i}$. We can compute $f\left(j, s_{1}, \ldots, s_{m^{*}}\right)$ given the values of $f\left(j-1, s_{1}^{\prime}, \ldots, s_{m^{*}}^{\prime}\right)$ for all $s_{1}^{\prime}, \ldots, s_{m^{*}}^{\prime} \in\left\{0, \ldots, \beta^{\prime}\right\}$ in time $O(|I|)$. As $\left|C^{*}\right| \leq \beta^{\prime}$, this means that all values $f\left(j, s_{1}, \ldots, s_{\left|C^{*}\right|}\right)$ and the bribery $\mathbf{t}^{*}$ itself can be computed in $O\left(\beta^{\prime \beta^{\prime}}|I|^{2}\right)$ time.

Greedy phase. In the last step, the algorithm iteratively constructs a set $V_{3}^{+}$using the following greedy procedure. We say that a voter is available if his shadow does not intersect the shadow of any of the voters already in $V_{3}^{+}$. Initially, the algorithm sets $V_{3}^{+}=\emptyset$. It then considers the missing colors one by one (in any order), and for each $i \in M$ it places any of the available voters from $R_{i}$ into $V_{3}^{+}$. As a final step, the algorithm picks one extra available vote, denoted by $v_{\text {extra }}$, from $R_{|M|}$. At the end of this procedure, the set $V_{3}^{+}$contains exactly one vote from $R_{i}$ for each missing color $i, i \neq|M|$, and exactly two votes from $R_{|M|}$, and has the property that the shadows of the voters in $V_{3}^{+}$are pairwise disjoint. We will now explain why it is possible to pick $|M|+1$ voters in this manner.

Briefly, the feasibility of our greedy procedure is implied by our choice of $r$. In more detail, observe that, whenever the algorithm has to pick the next voter to add to $V_{3}^{+}$, the total size of the shadows of the voters already in $V_{3}^{+}$does not exceed $\beta^{\prime}$; this is because each voter in $R_{i}$ has the same shadow size as $w_{i}$, and, by definition, we have $\sum_{i=1}^{k}\left|S\left(w_{i}\right)\right| \leq \beta^{\prime}$. Now, as the shadow of each voter in $R_{i}$ is a contiguous interval of length $\left|S\left(w_{i}\right)\right|$ in the order $\triangleleft$, there can be at most $\left|S\left(w_{i}\right)\right|$ voters in $R_{i}$ whose shadows contain a certain candidate. Thus there can be at most $\beta^{\prime}\left|S\left(w_{i}\right)\right| \leq \beta^{\prime 2}$ voters whose shadows contain any of the candidates in $\bigcup_{v \in V_{3}} S(v)$ and who are, hence, not available. Since $\left|R_{i}\right|=r$ for each $i \in M$, this means that for $r=\beta^{2}+1$ the algorithm can always choose an available voter.

We are now ready to define the output $\mathbf{t}_{\text {out }}$ of the algorithm: this is the bribery obtained by buying each voter in $V_{1}^{+} \cup V_{2}^{+} \cup V_{3}^{+}$, and then applying the bribery $\mathbf{t}^{*}$. The running time of this algorithm is $O\left(\beta^{\prime O\left(\beta^{\prime}\right)} n^{\lfloor 1 / \varepsilon\rfloor}|I|^{2}\right)$. We will now prove that it outputs a successful bribery of cost at most $(1+\varepsilon) B$ with probability at least $\beta^{\prime-\beta^{\prime}}$.

To see that the cost of $\mathbf{t}_{\text {out }}$ does not exceed $(1+\varepsilon) B$, observe first that in the branch of the algorithm that performs all the guesses correctly, the voters in 
$V_{1}^{+} \cup V_{2}^{+}$are exactly the voters in $V^{+} \backslash\left\{w_{i} \mid i \in M\right\}$. These voters are bought by $\mathbf{t}$, and therefore their price is present in the cost of $\mathbf{t}$ as well. Now, consider a voter $v \in V_{3}^{+}$. If his color is $i$, then his price does not exceed that of $w_{i}$. Hence, the total price of the voters in $V_{3}^{+} \backslash\left\{v_{\text {extra }}\right\}$ does not exceed the price of the voters $\left\{w_{i} \mid i \in M\right\}$, which is also included in the cost of $\mathbf{t}$. The cost of the bribery $\mathbf{t}^{*}$, which only decreases approval counts, is no greater than the amount spent by $\mathbf{t}$ on bribing the voters in $V^{-}$. Thus, we can conclude that the cost of $\mathbf{t}_{\text {out }}$ is at most the cost of $\mathbf{t}$ plus the price of $v_{\text {extra }}$. However, as $w_{|M|} \in V^{+} \backslash V_{1}^{+}$, we know that the price of $w_{|M|}$-and hence the price of $v_{\text {extra }}$-is less than $\varepsilon B$. This implies that the cost of $\mathbf{t}_{\text {out }}$ is at most $(1+\varepsilon) B$.

To complete the proof, we need to show that $\mathbf{t}_{\text {out }}$ succeeds in making $p$ a winner. Observe that, while the bribery $\mathbf{t}$ increases $p$ 's score by $\left|V^{+}\right|$, the bribery $\mathbf{t}_{\text {out }}$ increases $p$ 's score by $\left|V^{+}\right|+1$ because of the vote $v_{\text {extra }}$. This means that the total approval score of $p$ in $p \operatorname{sh}\left(C, V, \mathbf{t}_{\text {out }}\right)$ is $s^{*}+1$.

Let us fix a candidate $c \in C \backslash\{p\}$. First, assume that $c$ is not contained in the shadow of any of the voters in $V_{3}^{+}$. Then, by our definition of $s^{*}(c)$, after we buy the voters in $V_{1}^{+} \cup V_{2}^{+} \cup V_{3}^{+}$, the score of $c$ is exactly $s^{*}(c)$. Therefore, once we apply $\mathbf{t}^{*}$, the final score of $c$ in $p s h\left(C, V, \mathbf{t}_{\text {out }}\right)$ is at most $s^{*}$. Now, assume that $c$ is contained in the shadow of some voter in $V_{3}^{+}$. Since the shadows of the voters in $V_{3}^{+}$are pairwise disjoint, there is exactly one voter in $V_{3}^{+}$whose shadow contains $c$. This means that, after we buy the voters in $V_{1}^{+} \cup V_{2}^{+} \cup V_{3}^{+}$, the score of candidate $c$ is exactly $s^{*}(c)+1$, which implies that $c$ 's final score in $\operatorname{psh}\left(C, V, \mathbf{t}_{\text {out }}\right)$ is at most $s^{*}+1$. Hence, the score of every candidate $c \in C \backslash\{p\}$ in $\operatorname{psh}\left(C, V, \mathbf{t}_{\text {out }}\right)$ is at most $s^{*}+1$. It follows that $\mathbf{t}_{\text {out }}$ indeed makes $p$ a winner.

To derandomize the algorithm, we can use standard techniques relying on families of perfect hash functions, see [1]; note that randomization only occurs at the color-coding step.

\section{Conclusions and Future Work}

Our results show that shift bribery tends to be computationally easier than support bribery. However, in general, the power of these campaign management strategies is incomparable: one can construct examples of, e.g., Fallback elections where it is impossible to make someone a winner within a finite budget by shift bribery, but it is possible to do so by support bribery, or vice versa. Thus, both shift bribery and support bribery deserve to be studied in more detail.

An important contribution of this paper is the study of the parameterized version of support bribery, where the parameter is the total change in the approval counts. This natural parameterization leads to FPT algorithms for support bribery under two variants of the Fallback rule, as well as for SP-AV, for a large class of bribery cost functions. Also, we presented an approximation algorithm for the case of single-peaked preferences and unit costs that runs in FPT time with this parameterization. Finding other tractable parameterizations, or more generally, identifying further tractable cases (either in the classical, or in the parameterized sense) is an interesting direction for future research. The 
reader may wonder if it would make sense to study parameterized complexity of shift bribery. While for the voting rules considered in this paper shift bribery is polynomial-time solvable, for other rules it often is NP-complete [17]. Very recently, Bredereck et al. [9] gave a detailed parameterized study of shift bribery for several such voting rules.

\section{Acknowledgements}

A preliminary version of this paper was published in AAAI'11. We thank the AAAI and Algorithmica reviewers for their comments.

\section{References}

1. N. Alon, R. Yuster, and U. Zwick. Color-coding. Journal of the ACM, 42(4):844856, 1995.

2. J. Bartholdi, III and M. Trick. Stable matching with preferences derived from a psychological model. Operations Research Letters, 5(4):165-169, 1986.

3. D. Baumeister, G. Erdélyi, E. Hemaspaandra, L. Hemaspaandra, and J. Rothe. Computational aspects of approval voting. In J. Laslier and R. Sanver, editors, Handbook of Approval Voting, pages 199-251. Springer, 2010.

4. D. Baumeister, P. Faliszewski, J. Lang, and J. Rothe. Campaigns for lazy voters: Truncated ballots. In Proceedings of the 11th International Conference on Autonomous Agents and Multiagent Systems, pages 577-584, June 2012.

5. N. Betzler, A. Slinko, and J. Uhlmann. On the computation of fully proportional representation. Journal of Artificial Intelligence Research, 47:475-519, 2013.

6. S. Brams and R. Sanver. Critical strategies under approval voting: Who gets ruled in and ruled out. Electoral Studies, 25(2):287-305, 2006.

7. S. Brams and R. Sanver. Voting systems that combine approval and preference. In The Mathematics of Preference, Choice, and Order: Essays in Honor of Peter C. Fishburn, pages 215-237. Springer, 2009.

8. F. Brandt, M. Brill, E. Hemaspaandra, and L. Hemaspaandra. Bypassing combinatorial protections: Polynomial-time algorithms for single-peaked electorates. Journal of Artificial Intelligence Research, 53:439-496, 2015.

9. R. Bredereck, J. Chen, P. Faliszewski, A. Nichterlein, and R. Niedermeier. Prices matter for the parameterized complexity of shift bribery. In Proceedings of the 28th AAAI Conference on Artificial Intelligence, pages 1398-1404, 2014.

10. R. Bredereck, J. Chen, and G. Woeginger. Are there any nicely structured preference profiles nearby? In Proceedings of the $23 \mathrm{rd}$ International Joint Conference on Artificial Intelligence, pages 62-68, August 2013.

11. V. Conitzer. Eliciting single-peaked preferences using comparison queries. Journal of Artificial Intelligence Research, 35:161-191, 2009.

12. J. Doignon and J. Falmagne. A polynomial time algorithm for unidimensional unfolding representations. Journal of Algorithms, 16(2):218-233, 1994.

13. B. Dorn and I. Schlotter. Multivariate complexity analysis of swap bribery. Algorithmica, 64(1):126-151, 2012.

14. R. Downey and M. Fellows. Parameterized Complexity. Springer-Verlag, 1999.

15. J. Edmonds and R. Karp. Theoretical improvements in algorithmic efficiency for network flow problems. Journal of the ACM, 19(2):248-264, 1972. 
16. E. Elkind and P. Faliszewski. Approximation algorithms for campaign management. In Proceedings of the 6th International Workshop On Internet And Network Economics, pages 473-482. Springer-Verlag Lecture Notes in Computer Science \#6484, December 2010.

17. E. Elkind, P. Faliszewski, and A. Slinko. Swap bribery. In Proceedings of the 2nd International Symposium on Algorithmic Game Theory, pages 299-310. SpringerVerlag Lecture Notes in Computer Science \#5814, October 2009.

18. E. Elkind, P. Faliszewski, and A. Slinko. Swap bribery. Technical Report arXiv:0905.3885 [cs.GT], arXiv.org, May 2009.

19. E. Elkind, P. Faliszewski, and A. Slinko. On the role of distances in defining voting rules. In Proceedings of the 9th International Conference on Autonomous Agents and Multiagent Systems, pages 375-382. International Foundation for Autonomous Agents and Multiagent Systems, 2010.

20. G. Erdélyi, M. Fellows, J. Rothe, and L. Schend. Control complexity in Bucklin and Fallback voting: A theoretical analysis. Journal of Computer and System Sciences, 81(4):632-660, 2015.

21. G. Erdélyi, M. Fellows, J. Rothe, and L. Schend. Control complexity in Bucklin and Fallback voting: An experimental analysis. Journal of Computer and System Sciences, 81(4):661-670, 2015.

22. G. Erdélyi, M. Lackner, and A. Pfandler. The complexity of nearly single-peaked consistency. In Proceedings of the 27th AAAI Conference on Artificial Intelligence, pages 283-289, July 2013.

23. G. Erdélyi, M. Nowak, and J. Rothe. Sincere-strategy preference-based approval voting fully resists constructive control and broadly resists destructive control. Mathematical Logic Quarterly, 55(4):425-443, 2009.

24. B. Escoffier, J. Lang, and M. Öztürk. Single-peaked consistency and its complexity. In Proceedings of the 18th European Conference on Artificial Intelligence, pages 366-370. IOS Press, July 2008.

25. P. Faliszewski, E. Hemaspaandra, and L. Hemaspaandra. How hard is bribery in elections? Journal of Artificial Intelligence Research, 35:485-532, 2009.

26. P. Faliszewski, E. Hemaspaandra, and L. Hemaspaandra. Using complexity to protect elections. Communications of the ACM, 53(11):74-82, 2010.

27. P. Faliszewski, E. Hemaspaandra, and L. Hemaspaandra. The complexity of manipulative attacks in nearly single-peaked electorates. Artificial Intelligence, 207:6999, 2014.

28. P. Faliszewski, E. Hemaspaandra, and L. Hemaspaandra. Weighted electoral control. Journal of Artificial Intelligence Research, 52:507-542, 2015.

29. P. Faliszewski, E. Hemaspaandra, L. Hemaspaandra, and J. Rothe. The shield that never was: Societies with single-peaked preferences are more open to manipulation and control. Information and Computation, 209(2):89-107, 2011.

30. P. Faliszewski, Y. Reisch, J. Rothe, and L. Schend. Complexity of manipulation, bribery, and campaign management in Bucklin and fallback voting. Autonomous Agents and Multi-Agent Systems, 2015. To appear.

31. P. Faliszewski and J. Rothe. Control and bribery in voting. In F. Brandt, V. Conitzer, U. Endriss, J. Lang, and A. D. Procaccia, editors, Handbook of Computational Social Choice, chapter 7. Cambridge University Press, 2015.

32. M. Fellows, D. Hermelin, F. Rosamond, and S. Vialette. On the parameterized complexity of multiple-interval graph problems. Theoretical Computer Science, 410:53-61, 2009.

33. J. Flum and M. Grohe. Parameterized Complexity Theory. Springer-Verlag, 2006. 
34. E. Hemaspaandra, L. Hemaspaandra, and J. Rothe. Anyone but him: The complexity of precluding an alternative. Artificial Intelligence, 171(5-6):255-285, 2007.

35. H. Kuhn. The Hungarian method for the assignment problem. Naval Research Logistics Quarterly, 2(1-2):83-97, 1955.

36. J. Laslier and K. Van der Straeten. A live experiment on approval voting. Experimental Economics, 11(1):97-105, 2008.

37. T. Magrino, R. Rivest, E. Shen, and D. Wagner. Computing the margin of victory in IRV elections. In Electronic Voting Technology Workshop/Workshop on Trushworthy Elections, August 2011.

38. C. Menton. Normalized range voting broadly resists control. Theory of Computing Systems, 53(4):507-531, 2013.

39. M. Naor, L. Schulman, and A. Srinivasan. Splitters and near-optimal derandomization. In Proceedings of the 36th IEEE Symposium on Foundations of Computer Science, pages 182-191, 1995.

40. R. Niedermeier. Invitation to Fixed-Parameter Algorithms. Oxford University Press, 2006.

41. K. Van der Straeten, J. Laslier, N. Sauger, and A. Blais. Strategic, sincere, and heuristic voting under four election rules: An experimental study. Social Choice and Welfare, 35(3):435-472, 2010.

42. É. Tardos. A strongly polynomial minimum cost circulation algorithm. Combinatorica, 5(3):247-255, 1985.

43. T. Walsh. Uncertainty in preference elicitation and aggregation. In Proceedings of the 22nd AAAI Conference on Artificial Intelligence, pages 3-8. AAAI Press, July 2007.

44. L. Xia. Computing the margin of victory for various voting rules. In Proceedings of the 13th ACM Conference on Electronic Commerce, pages 982-999, June 2012.

45. L. Xia and V. Conitzer. Determining possible and necessary winners given partial orders. Journal of Artificial Intelligence Research, 41:25-67, 2011.

46. L. Xia, M. Zuckerman, A. Procaccia, V. Conitzer, and J. Rosenschein. Complexity of unweighted manipulation under some common voting rules. In Proceedings of the 21st International Joint Conference on Artificial Intelligence, pages 348-353. AAAI Press, July 2009.

\section{A Destructive Support Bribery}

In this appendix, we briefly discuss destructive support bribery. In the destructive variant of the problem, the goal is not to ensure a preferred candidate's victory, but to prevent a despised candidate from winning. In contrast to our hardness results for constructive support bribery, we can show that destructive support bribery is easy for SP-AV, simplified Fallback voting, and Fallback voting.

Theorem 9. DESTRUCTIVE SUPPORT BRIBERY is in $\mathrm{P}$ for each of $S P-A V$, simplified Fallback voting, and Fallback voting.

Proof. For of each of SP-AV, simplified Fallback voting, and Fallback voting, we use the same strategy and compute certain functions defined below using dynamic programming. 
Let $E=(C, V)$ be an election, where $C=\left\{d, c_{1}, \ldots, c_{m-1}\right\}$ and $V=$ $\left(v^{1}, \ldots, v^{n}\right)$ is a collection of voters (each voter $v^{i}$ has preference order $\succ^{i}$ and approval count $\ell^{i}$ ). We are also given support bribery cost functions $\Sigma=$ $\left(\sigma^{1}, \ldots, \sigma^{n}\right)$ for all voters. The outline of our algorithm, same for each of our voting rules, is as follows:

1. For each candidate $c \in C \backslash\{d\}$ compute the lowest cost of ensuring that $c$ prevents $d$ from being a winner.

2. Output the minimum of the costs computed in the previous step.

Naturally, the exact meaning of "c prevents $d$ from being a winner" is different for each of our voting rules. For SP-AV it means that (a) $c$ has more approvals in total than $d$ has. For simplified Fallback voting it means that either $\left(\mathrm{a}^{\prime}\right) c$ has more approvals in total than $d$ and $d$ does not win in any round, or (b) $c$ wins in some round $t$ and $d$ does not win in round $t$. In case of Fallback voting, we need either $\left(\mathrm{a}^{\prime}\right)$ or $\left(\mathrm{b}^{\prime}\right) c$ wins in some round $t, d$ does not win in round $t-1$, and $c$ has more $t$-approval points than $d$.

Let us fix a candidate $c \in C \backslash\{d\}$. To describe algorithms computing a lowestcost support bribery for each of the above conditions, for each $k, i, j$ in $\{0, \ldots, n\}$ and for each $t$ in $\{1, \ldots, m\}$ we define $f_{t}(k, i, j)$ to be the cost of a minimum-cost support bribery ensuring that exactly $i$ voters in $V_{k}=\left\{v^{1}, \ldots, v^{k}\right\}$ approve $c$ and rank her in top $t$ positions and exactly $j$ voters in $V_{k}$ approve $d$ and rank her in top $t$ positions. It is easy to verify that for each $t$ and each $k, i, j$ in this range we can easily compute $f_{t}(k, i, j)$ in polynomial time using standard dynamic programming techniques.

Now, it is easy to see that the lowest cost of ensuring that $c$ has more approvals than $d$ (condition (a)) is exactly $\min \left\{f_{m}(n, i, j) \mid i>j\right\}$. Similarly, the minimum cost of ensuring that condition $\left(\mathrm{a}^{\prime}\right)$ holds is $\min \left\{f_{m}(n, i, j) \mid i>j, j<\right.$ $\left.\left\lfloor\frac{n}{2}\right\rfloor+1\right\}$. The lowest cost of ensuring that either $c$ wins in an earlier round than $d$ or $c$ wins in some round but $d$ does not (condition (b)) is

$$
\min \left\{f_{t}(n, i, j) \mid i \geq\left\lfloor\frac{n}{2}\right\rfloor+1, j<\left\lfloor\frac{n}{2}\right\rfloor+1,1 \leq t \leq m\right\} .
$$

To deal with the case of classic Fallback voting and compute the lowest cost of ensuring condition $\left(\mathrm{b}^{\prime}\right)$, we have to modify our family of functions $f_{t}$ a little. For each $k, i, j, r$ in $\{0, \ldots, n\}$ and each $t$ in $\{1, \ldots, m\}$, let $f_{t}^{\prime}(k, i, j, r)$ be the cost of a lowest-cost support bribery ensuring that exactly $i$ voters in $V_{k}=\left\{v^{1}, \ldots, v^{k}\right\}$ approve $c$ and rank her in top $t$ positions, exactly $j$ voters in $V_{k}$ approve $d$ and rank her in top $t$ positions, and exactly $r$ voters in $V_{k}$ approve $d$ and rank her in top $t-1$ positions. By construction, each function $f_{t}^{\prime}$ is computable in polynomial time using standard dynamic programming techniques. Using these functions, we can compute the lowest cost of making $c$ win in some round where she has more approvals than $d$ does, while also making sure that $d$ does not win in the previous round (condition $\left(\mathrm{b}^{\prime}\right)$ ):

$$
\min \left\{f_{t}^{\prime}(n, i, j, r) \mid i \geq\left\lfloor\frac{n}{2}\right\rfloor+1, i>j, r<\left\lfloor\frac{n}{2}\right\rfloor+1,1 \leq t \leq m\right\} .
$$


These observations show that destructive support bribery is in $\mathrm{P}$ for each of SP-AV, simplified Fallback voting, and Fallback voting.

Not much is known about destructive shift bribery, where the briber can ask the voters to demote the despised candidate $d$ in order to prevent her from winning the elections; we propose algorithmic analysis of this form of bribery as a topic for future work. Additional motivation for the study of destructive shift bribery is provided by recent work that suggests some very interesting applications of this concept $[37,44]$. 